\title{
Optimal Control and Geodesics on Quadratic Matrix Lie Groups
}

\author{
Anthony M. Bloch - Peter E. Crouch • \\ Jerrold E. Marsden • Amit K. Sanyal
}

Received: 17 January 2007 / Revised: 5 December 2007 / Accepted: 28 January 2008 / Published online: 28 February 2008

(C) SFoCM 2008

\begin{abstract}
The purpose of this paper is to extend the symmetric representation of the rigid body equations from the group $\mathrm{SO}(n)$ to other groups. These groups are matrix subgroups of the general linear group that are defined by a quadratic matrix identity. Their corresponding Lie algebras include several classical semisimple matrix Lie algebras. The approach is to start with an optimal control problem on these groups that generates geodesics for a left-invariant metric. Earlier work by Bloch, Crouch, Marsden, and Ratiu defines the symmetric representation of the rigid body equations, which is obtained by solving the same optimal control problem in the particular case of the Lie group $\mathrm{SO}(n)$. This paper generalizes this symmetric representation to a
\end{abstract}

Dedicated to Professor Arieh Iserles on the Occasion of his Sixtieth Birthday.

Communicated by Hans Munthe-Kaas.

A.M. Bloch $(\bowtie)$

Department of Mathematics, University of Michigan, Ann Arbor, MI 48109, USA

e-mail: abloch@umich.edu

P.E. Crouch

Department of Electrical Engineering, University of Hawaii, Honolulu, HI 96822, USA

e-mail: pcrouch@hawaii.edu

J.E. Marsden

Department of Control and Dynamical Systems, California Institute of Technology, Pasadena, CA 91125, USA

e-mail: jmarsden@caltech.edu

\section{A.K. Sanyal}

Department of Mechanical Engineering, University of Hawaii, Honolulu, HI 96822, USA

e-mail: aksanyal@hawaii.edu 
wider class of matrix groups satisfying a certain quadratic matrix identity. We consider the relationship between this symmetric representation of the generalized rigid body equations and the generalized rigid body equations themselves. A discretization of this symmetric representation is constructed making use of the symmetry, which in turn give rise to numerical algorithms to integrate the generalized rigid body equations for the given class of matrix Lie groups.

Keywords Geodesics · Optimal control · Generalized rigid body equations

Mathematics Subject Classification (2000) 34H05 · 70E40 - 49K15

\section{Introduction}

This paper treats an optimal control problem on some subgroups of the general and special linear group that satisfy a quadratic matrix identity. These groups include certain classical Lie groups and their corresponding Lie algebras.

The extremal solutions to this optimal control problem are obtained as geodesic flows on the group with respect to a given positive definite metric, and are represented on the product space of the group with itself. These equations generalize the symmetric representation of the rigid-body equations given in [3] and some properties of rigid body dynamics.

Variational and optimal control problems on Lie groups and symmetric spaces have been treated before in the literature. The Euler equations on the $n$-dimensional rigid body have been treated, for example, in [7, 13, 17], besides the symmetric representation in [3].

The symmetric representation of the generalized rigid body equations on the $n$-dimensional proper orthogonal group $\mathrm{SO}(n)$ (whose Lie algebra is denoted $\mathfrak{s o}(n)$ ) is the set of equations

$$
\dot{Q}=Q \Omega ; \quad \dot{P}=P \Omega .
$$

The notation in these equations is as follows: The matrices $Q$ and $P$ are the dynamical variables, where $Q \in \mathrm{SO}(n)$ denotes the configuration of the body and $P$ is a costate variable associated with the optimal control problem associated to these equations. For these equations to make sense as first order equations on $\mathrm{SO}(n) \times \mathrm{SO}(n)$, one needs to specify how $\Omega=Q^{-1} \dot{Q} \in \mathfrak{s o}(n)$, the body angular velocity is a function of $Q$ and $P$, and thereby find a way to transform these equations to the usual Euler equations for the $n$-dimensional rigid body. This will be explained in the body of the paper below.

In this paper, we generalize these equations as well as the Euler equations for some matrix subgroups of the general linear group that are defined by a quadratic matrix identity. We introduce these matrix subgroups of GL( $n)$ and give some of their properties in Sect. 2. We introduce the optimal control problem on these groups in Sect. 3, and obtain the extremal solutions to this optimal control problem as a flow on the product group. In Sect. 3, we also obtain a map that transforms the symmetric representation to the Euler representation of the geodesic flows. In Sect. 4, we analyze 
the state space of extremal solutions in the symmetric representation, and introduce a symplectic submanifold in the product group that is invariant with respect to the extremal flows. In Sect. 5, we present some results on the discrete version of the optimal control problem, and obtain the symmetric representation of the discrete extremal flows. Section 6 presents some conclusions and discusses planned extensions of this work.

\section{Quadratic Matrix Groups}

We consider quadratic matrix groups of the form

$$
G:=\left\{g \in \mathrm{GL}(n) \mid g^{\top} J g=J\right\},
$$

where $g^{\top}$ is the transpose of the $n \times n$ matrix $g, J^{2}=\alpha I_{n}$ and $J^{\top}=\alpha J$ for $\alpha=$ \pm 1 . Taking determinants in the relation $g^{\top} J g=J$ in (2.1), we note that for $g \in G$, $\operatorname{det} g= \pm 1$.

This class of groups includes standard classical groups of interest including the symplectic group and $\mathrm{O}(p, q)$ (see below), and enables one to generalize the symmetric representation of the rigid body flows discussed in [3] to this class. This class of matrix groups gives matrix representations of linear transformations on $\mathbb{R}^{n}$ that leave the following symmetric, bilinear form invariant:

$$
f(x, y)=x^{\top} J y, \quad x, y \in \mathbb{R}^{n} .
$$

Thus, if $g \in G$, then

$$
f(g x, g y)=x^{\top} g^{\top} J g y=x^{\top} J y=f(x, y) .
$$

Since $J$ is not singular, the bilinear form $f(x, y)$ is nondegenerate.

The conditions following the definition (2.1) imply that $J^{\top}=J^{-1}=\alpha J$. Some examples of matrix groups covered by this definition are given below.

\section{Examples}

1. Choosing $J=I_{n}$ (the $n \times n$ identity matrix) and $\alpha=1$, then $G=\mathrm{O}(n)$; this is somewhat different from considering the component of the identity, namely the case of $\mathrm{SO}(n)$, which was treated in [3].

2. If $n=2 m$ is even and

$$
J=\left[\begin{array}{cc}
0 & I_{m} \\
-I_{m} & 0
\end{array}\right],
$$

then the above definition gives the group $\operatorname{Sp}(2 m)$ of $2 m \times 2 m$ symplectic matrices. We remark that the paper [4] considers a related class of flows on symplectic groups with $J$ generalized to be an arbitrary skew-symmetric invertible matrix. Interestingly for integrability in that case, one typically does not use the case of canonical $J$. 
3. If $n=p+q$ and

$$
J=\left[\begin{array}{cc}
I_{p} & 0 \\
0 & -I_{q}
\end{array}\right],
$$

then the above definition gives the group $\mathrm{O}(p, q)$ of matrices that leave the nondegenerate, symmetric, bilinear form of signature $(p, q)$ in $\mathbb{R}^{n}$ invariant.

The Lie algebra of the group $G$ is given by

$$
\mathfrak{g}=\left\{X \in \mathfrak{g l}(n) \mid X^{\top} J+J X=0\right\} .
$$

Note that for $X \in \mathfrak{g}, J^{-1} X^{\top} J=-X$ and so

$$
\operatorname{Trace}(X)=-\operatorname{Trace}\left(J^{-1} X^{\top} J\right)=-\operatorname{Trace}\left(X^{\top}\right)=-\operatorname{Trace}(X)
$$

and so $\operatorname{Trace}(X)=0$. Therefore, the Lie algebra $\mathfrak{g}$ of $G$ is a subalgebra of $\mathfrak{s l}(n)$. The following statement constructs an element of the Lie algebra $\mathfrak{g}$ from a given element in the group $G$.

Lemma 2.1 If $g \in G$, then $g^{\top} \in G$ and $g-g^{-1} \in \mathfrak{g}$.

Proof Taking the matrix inverse of the relation $g^{\top} J g=J$ and using the fact that $J^{-1}=\alpha J$, we get

$$
\begin{gathered}
J^{-1}=g^{-1} J^{-1}\left(g^{\top}\right)^{-1} \Rightarrow J=g^{-1} J\left(g^{\top}\right)^{-1} \\
\Rightarrow \quad\left(g^{\top}\right)^{-1} \in G \Rightarrow g^{\top} \in G .
\end{gathered}
$$

If $g \in G$, then

$$
\left(g-g^{-1}\right)^{\top} J+J\left(g-g^{-1}\right)=\left(g^{\top} J-J g^{-1}\right)+\left(J g-\left(g^{-1}\right)^{\top} J\right) .
$$

But from the relation $g^{\top} J g=J$, we get

$$
g^{\top} J=J g^{-1} \quad \text { and } \quad J g=\left(g^{\top}\right)^{-1} J .
$$

Hence, we have

$$
\left(g-g^{-1}\right)^{\top} J+J\left(g-g^{-1}\right)=0,
$$

and thus $g-g^{-1} \in \mathfrak{g}$.

The following statement combined with the previous statement leads to a trace orthogonal decomposition of the group $G$.

Lemma 2.2 If $g \in G$ and $U \in \mathfrak{g}$, then

$$
\operatorname{Trace}(g U)=-\operatorname{Trace}\left(g^{-1} U\right) .
$$


Proof Using the identities $J^{\top}=\alpha J=J^{-1}$ and $U^{\top} J+J U=0$, we obtain

$$
\begin{aligned}
\operatorname{Trace}(g U) & =-\operatorname{Trace}\left(g J^{-1} U^{\top} J\right)=-\operatorname{Trace}\left(J g J^{-1} U^{\top}\right) \\
& =-\operatorname{Trace}\left(U\left(J^{-1}\right)^{\top} g^{\top} J^{\top}\right)=-\operatorname{Trace}\left(\left(J^{-1}\right)^{\top} g^{\top} J^{\top} U\right) \\
& =-\operatorname{Trace}\left(\alpha^{-1} J^{-1} g^{\top} \alpha J U\right)=-\operatorname{Trace}\left(J^{-1} g^{\top} J U\right)=-\operatorname{Trace}\left(g^{-1} U\right),
\end{aligned}
$$

using the identity $g^{\top} J g=J$ in the last step.

Thus, for any $g \in G$, we have

$$
\operatorname{Trace}\left(\left(g+g^{-1}\right) U\right)=0
$$

and hence,

$$
g=\frac{1}{2}\left(g+g^{-1}\right)+\frac{1}{2}\left(g-g^{-1}\right)
$$

provides a trace orthogonal decomposition of the group $G$. In the special case when $J=I_{n}, \alpha=1, G=\mathrm{SO}(n)$, the above decomposition is the standard symmetric plus skew-symmetric decomposition:

$$
Q=\frac{1}{2}\left(Q+Q^{\top}\right)+\frac{1}{2}\left(Q-Q^{\top}\right)
$$

where $Q \in \mathrm{SO}(n), Q+Q^{\top}$ is symmetric, and $Q-Q^{\top} \in \mathfrak{s o}(n)$ is skew-symmetric.

Note that if $\Lambda=g+g^{-1}$ such that $g \in G$, then $\Lambda$ satisfies $J \Lambda-\Lambda^{\top} J=0$. This follows since if $\Lambda=g+g^{-1}$ for some $g \in G$, then

$$
J \Lambda=J\left(g+g^{-1}\right)=J g+J g^{-1}=\left(g^{\top}\right)^{-1} J+g^{\top} J=\left(g+g^{-1}\right)^{\top} J=\Lambda^{\top} J .
$$

In the next section, we will show how such a matrix $\Lambda$ can be used to construct a metric for $\mathfrak{g}$.

As a corollary of Lemma 2.2, we obtain the following result.

Corollary 2.3 If $g \in G$ and $U, V \in \mathfrak{g}$, then

$$
\operatorname{Trace}(g V U)=\operatorname{Trace}\left(g^{-1} U V\right)
$$

Proof We differentiate the identity (2.2) with respect to $g$ in the direction $g V$. This gives the identity:

$$
\operatorname{Trace}(g V U)=\operatorname{Trace}\left(g^{-1} g V g^{-1} U\right),
$$

which is equivalent to

$$
\operatorname{Trace}(g V U)=\operatorname{Trace}\left(g^{-1} U V\right)
$$

which gives the stated result. 
These algebraic relations will be helpful in the analysis of the geodesic flows in the following section. In particular, they are useful in formulating a symmetric representation of the geodesic flows analogous to that for the generalized rigid body problem on $\operatorname{SO}(n)$.

\section{The Optimal Control Problem}

This section recalls how to obtain geodesic flows using the maximum principle of optimal control theory (as in [2, 8, 12]). Using this, an optimal control problem for the quadratic matrix group $G$ is introduced.

\subsection{Optimal Control Problem on Quadratic Matrix Lie Groups}

Let $\Sigma: \mathfrak{g} \rightarrow \mathfrak{g}$ be a symmetric positive definite operator with respect to the inner product given by the trace pairing (which defines an inner product on both $\mathfrak{g}$ and $\mathfrak{g l}(n))$ :

$$
\langle A, B\rangle=\operatorname{Trace}\left(A^{\top} B\right) .
$$

The optimal control problem of interest here is the following:

Optimal Control Problem on $G$ Minimize the integral

$$
\int_{0}^{T} \frac{1}{4}\langle U, \Sigma(U)\rangle \mathrm{d} t
$$

over all curves $Q(t) \in G$ with $t \in[0, T]$ and with fixed endpoints $Q(0)=Q_{0} \in G$ and $Q(T)=Q_{T} \in G$, and where $U$ is defined by $\dot{Q}=Q U$, so that $U \in \mathfrak{g}$.

Since $\Sigma$ defines a metric on $\mathfrak{g}$ given by

$$
\langle U, V\rangle_{\Sigma}=\langle U, \Sigma(V)\rangle=\langle\Sigma(U), V\rangle
$$

the problem (3.2) can be regarded as an optimal control problem with $U \in \mathfrak{g}$ thought of as the control. This optimal control viewpoint for this problem leads to an interesting form of the corresponding geodesic equations as is developed in this section.

The point is that the problem (3.2) may be viewed as a geodesic problem in several ways. If one directly seeks the curve $Q(t) \in G$, then one is clearly seeking a geodesic on $Q(t) \in G$ relative to the left invariant kinetic energy metric given at the identity by (3.3). Alternatively, one can relax the "constraints" $U=Q^{-1} \dot{Q}$ and put corresponding constraints on the allowed variations of $U$, which would result in the Euler-Poincaré variational formulation of the problem (see, for instance, [14], Chap. 13). Yet another way to view this problem is via the Pontryagin maximum principle, which is closely related to the Hamilton-Pontryagin principle (see [20]); we do this in the next subsection below.

The following result constructs an interesting class of positive definite symmetric mappings $\Sigma: \mathfrak{g} \rightarrow \mathfrak{g}$; it gives an explicit form of the operator which generalizes the form used in the case of $\mathrm{SO}(n)$. However, the theory below holds for general positive definite symmetric operators $\Sigma$. 
Proposition 3.1 Let $\Lambda$ be a real $n \times n$ matrix satisfying $\Lambda^{\top} J=J \Lambda$ and be such that the symmetric matrix $\Lambda+\Lambda^{\top}$ is positive definite. Then $\Sigma: \mathfrak{g} \rightarrow \mathfrak{g}$ defined by

$$
\Sigma(U)=\frac{1}{2}\left[U\left(\Lambda+\Lambda^{\top}\right)+\left(\Lambda+\Lambda^{\top}\right) U\right]
$$

is a symmetric positive definite operator.

Proof We first show that $\Lambda$ also satisfies $\Lambda J=J \Lambda^{\top}$. This is shown as follows:

$$
\Lambda J=\left(J^{\top} \Lambda^{\top} J\right) J=J^{\top} \Lambda^{\top} J^{2}=\alpha J^{\top} \Lambda^{\top}=J \Lambda^{\top} .
$$

If $\Sigma$ is as defined in (3.4), then

$$
\begin{aligned}
& (U \Lambda+\Lambda U)^{\top} J+J(U \Lambda+\Lambda U) \\
& =\Lambda^{\top} U^{\top} J+U^{\top} \Lambda^{\top} J+J U \Lambda+J \Lambda U \\
& =\Lambda^{\top} U^{\top} J+\Lambda^{\top} J U+U^{\top} J \Lambda+J U \Lambda \\
& =\Lambda^{\top}\left(U^{\top} J+J U\right)+\left(U^{\top} J+J U\right) \Lambda=0,
\end{aligned}
$$

and

$$
\begin{aligned}
& \left(U \Lambda^{\top}+\Lambda^{\top} U\right)^{\top} J+J\left(U \Lambda^{\top}+\Lambda^{\top} U\right) \\
& =\Lambda U^{\top} J+U^{\top} \Lambda J+J U \Lambda^{\top}+J \Lambda^{\top} U \\
& =\Lambda U^{\top} J+\Lambda J U+U^{\top} J \Lambda^{\top}+J U \Lambda^{\top} \\
& =\Lambda\left(U^{\top} J+J U\right)+\left(U^{\top} J+J U\right) \Lambda^{\top}=0 .
\end{aligned}
$$

This shows that indeed $\Sigma: \mathfrak{g} \rightarrow \mathfrak{g}$. Symmetry and positive definiteness is shown by the following calculation:

$$
\begin{aligned}
\langle U, \Sigma(V)\rangle & =\frac{1}{2} \operatorname{Trace}\left(U^{\top}\left(V\left(\Lambda+\Lambda^{\top}\right)+\left(\Lambda+\Lambda^{\top}\right) V\right)\right) \\
& =\frac{1}{2} \operatorname{Trace}\left(V\left(\Lambda+\Lambda^{\top}\right) U^{\top}-U^{\top}\left(\Lambda+\Lambda^{\top}\right) V\right) \\
& =\frac{1}{2} \operatorname{Trace}\left(\left(V \Lambda U^{\top}+U^{\top} \Lambda V\right)+\left(V \Lambda^{\top} U^{\top}+U^{\top} \Lambda^{\top} V\right)\right) \\
& =\frac{1}{2} \operatorname{Trace}\left(\left(V \Lambda U^{\top}+J U \Lambda^{\top} V^{\top} J^{\top}\right)+\left(V \Lambda^{\top} U^{\top}+J U \Lambda^{\top} J^{\top}\right)\right) \\
& =\frac{1}{2} \operatorname{Trace}\left(2 V \Lambda U^{\top}+2 V \Lambda^{\top} U^{\top}\right)=\operatorname{Trace}\left(V\left(\Lambda+\Lambda^{\top}\right) U^{\top}\right),
\end{aligned}
$$

which is symmetric and positive definite since $\Lambda+\Lambda^{\top}$ is positive definite.

Thus, if $\Lambda$ is $J$-symmetric (i.e., $\Lambda^{\top} J=J \Lambda$ ) and positive definite, then $\Sigma: \mathfrak{g} \rightarrow \mathfrak{g}$ as defined by (3.4) is a symmetric positive definite operator. 
Example For the case $G=\operatorname{Sp}(2 m)$, let

$$
\Lambda=\left[\begin{array}{cc}
\Lambda_{0} & 0 \\
0 & \Lambda_{0}
\end{array}\right], \quad \text { where } \Lambda_{0}^{\top}=\Lambda_{0} .
$$

Recalling that for this group, $J=\left[\begin{array}{cc}0 & I_{m} \\ -I_{m} & 0\end{array}\right]$, we see that $\Lambda$ is $J$-symmetric; that is,

$$
\Lambda^{\top} J=\left[\begin{array}{cc}
0 & \Lambda_{0} \\
-\Lambda_{0} & 0
\end{array}\right]=J \Lambda .
$$

The condition that $\Lambda+\Lambda^{\top}$ be positive definite that is required in Proposition 3.1 is equivalent to the condition that $\Lambda_{0}$ is positive definite. This gives a choice for $\Sigma$ when $G=\operatorname{Sp}(2 m)$. Interestingly, this choice of $\Lambda$ works even in the case that $G=\mathrm{O}(m, m)$, as can be easily verified using Proposition 3.1.

The remainder of this section determines the extremal equations for the optimal control problem (3.2). To do this, we first relate the problem to a Hamiltonian flow on the symplectic manifold $\mathfrak{g l}(n) \times \mathfrak{g l}(n)$ with the canonical symplectic form:

$$
\Omega_{\text {can }}\left(\left(X_{1}, Y_{1}\right),\left(X_{2}, Y_{2}\right)\right)=\left\langle Y_{2}, X_{1}\right\rangle-\left\langle Y_{1}, X_{2}\right\rangle .
$$

We then demonstrate that this flow leaves $G \times G$ invariant, and hence naturally restricts. We further show that on an open submanifold $S \subset G \times G$ where $\Omega_{\text {can }}$ restricts to a symplectic form, the flow is also Hamiltonian. Moreover, this restricted flow on $S \subset G \times G$ is locally equivalent to the generalized Euler equations in $G \times \mathfrak{g}$ (or equivalently in $T^{\star} G$ ) as we show in this section and the next.

\subsection{Extremal Flow in Symmetric Representation}

In this section, we consider the geodesic flow as a constrained optimization problem and the corresponding equations are determined by using Pontryagin's maximum principle (see, for example, [2]). We begin by considering a related optimal control problem.

\section{Optimal Control Problem in $\mathfrak{g l}(\boldsymbol{n})$ Minimize}

$$
\int_{0}^{T} \frac{1}{4}\langle U, \Sigma(U)\rangle \mathrm{d} t
$$

over all curves $Q(t) \in \mathfrak{g l}(n)$ with $t \in[0, T]$ and with fixed endpoints $Q(0)=Q_{0}$ and $Q(T)=Q_{T}$, and where $U$ satisfies the constraint $\dot{Q}=Q U$ with $U \in \mathfrak{g}$.

It will then be shown that one can restrict to the domain of interest, namely the quadratic matrix group $G$. Let $P \in \mathfrak{g l}(n)$ denote the costate variable (consisting of Lagrange multipliers) used to impose the kinematic constraint $\dot{Q}=Q U$. The Hamiltonian for the optimal control problem (3.6) is then defined as

$$
H(P, Q, U)=\langle P, Q U\rangle-\frac{1}{4}\langle U, \Sigma(U)\rangle=\left\langle Q^{\top} P, U\right\rangle-\frac{1}{4}\langle U, \Sigma(U)\rangle
$$


Since $(Q, P) \in \mathfrak{g l}(n) \times \mathfrak{g l}(n)$ and $U$ is an element of $\mathfrak{g}, H$ is a mapping:

$$
H: \mathfrak{g l}(n) \times \mathfrak{g l}(n) \times \mathfrak{g} \rightarrow \mathbb{R} .
$$

The following result will be established using Pontryagin's maximum principle.

Proposition 3.2 The optimal trajectories of the optimal control problem (3.6) are given by the following system of Hamiltonian equations on the symplectic manifold $\left(\mathfrak{g l}(n) \times \mathfrak{g l}(n), \Omega_{\text {can }}\right):$

$$
\begin{aligned}
& \dot{Q}=Q U, \quad Q \in \mathfrak{g l}(n), \\
& \dot{P}=-P U^{\top}, \quad P \in \mathfrak{g l}(n), \\
& U=\Sigma^{-1}\left(Q^{\top} P-\alpha J P^{\top} Q J\right) \in \mathfrak{g} .
\end{aligned}
$$

Equation (3.9) can be interpreted as a projection of $Q^{\top} P$ from $\mathfrak{g l}(n)$ to $\mathfrak{g}$. To show that this is indeed a projection, we use the following lemma.

Lemma 3.3 The map $\mathrm{P}: \mathfrak{g l}(n) \rightarrow \mathfrak{g}$ given by

$$
\mathrm{P}(A)=\frac{1}{2}\left(A-\alpha J A^{\top} J\right),
$$

is a projection. Thus, (3.9) can be written as $U=2 \Sigma^{-1} \mathrm{P}\left(Q^{\top} P\right)$.

Proof of Lemma We compute

$$
\begin{aligned}
\mathrm{P}^{2}(A)=\mathrm{P}(\mathrm{P}(A)) & =\frac{1}{4}\left[A-\alpha J A^{\top} J-\alpha J\left(A-\alpha J A^{\top} J\right)^{\top} J\right] \\
& =\frac{1}{4}\left[A-\alpha J A^{\top} J-\alpha J\left(A^{\top}-\alpha J^{\top} A J^{\top}\right) J\right] \\
& =\frac{1}{4}\left[A-2 \alpha J A^{\top} J+J J^{\top} A J^{\top} J\right] \\
& =\frac{1}{2}\left[A-\alpha J A^{\top} J\right]=\mathrm{P}(A),
\end{aligned}
$$

since $J J^{\top}=J^{\top} J=\alpha J^{2}=I_{n}$. This proves the result.

Now we are ready to prove the proposition.

Proof of Proposition From the necessary conditions for optimality, we get

$$
\dot{Q}=\operatorname{grad}_{P} H(P, Q, U)=Q U,
$$

where we employ the gradient operator with respect to the inner product on $\mathfrak{g l}(n)$, $\langle\cdot, \cdot\rangle$ given by (3.1). The second of (3.8) is obtained similarly as

$$
\dot{P}=-\operatorname{grad}_{Q} H(P, Q, U)=-\operatorname{grad}_{Q}\langle P, Q U\rangle=-\operatorname{grad}_{Q}\left\langle P U^{\top}, Q\right\rangle=-P U^{\top} .
$$


To complete the proof, we need to specify the optimal control $U \in \mathfrak{g}$. This is obtained from the maximum principle (see p. 336 of [2]) as follows:

$$
\max _{U \in \mathfrak{g}} H(Q(t), P(t), U)=H\left(Q(t), P(t), U^{*}(t)\right),
$$

where $U^{*}(t)$ is the optimal control function; in fact, we show below that (3.11) does result in a unique specification for $U^{*}$. Thus, in this case where $H$ is defined by (3.7), we must perform the following optimization problem:

$$
\max _{U}\left(\left\langle Q^{\top} P, U\right\rangle-\frac{1}{4}\langle U, \Sigma(U)\rangle+\frac{1}{2}\left\langle\Pi, U^{\top} J+J U\right\rangle\right),
$$

where $\Pi$ is a Lagrange multiplier for the constraint that $U$ lie in $\mathfrak{g}$; that is, $U^{\top} J+$ $J U=0$. Now

$$
\left(U^{\top} J+J U\right)^{\top}=U^{\top} J^{\top}+J^{\top} U=\alpha\left(U^{\top} J+J U\right)
$$

So we choose $\Pi$ to satisfy $\Pi^{\mathrm{T}}=\alpha \Pi$. Thus,

$$
\begin{aligned}
\left\langle\Pi, U^{\top} J+J U\right\rangle & =\left\langle\Pi J^{\top}, U^{\top}\right\rangle+\left\langle J^{\top} \Pi, U\right\rangle \\
& =\left\langle J \Pi^{\top}, U\right\rangle+\left\langle J^{\top} \Pi, U\right\rangle=2 \alpha\langle J \Pi, U\rangle .
\end{aligned}
$$

Therefore, we have

$$
\frac{1}{2}\left\langle\Pi, U^{\top} J+J U\right\rangle=\alpha\langle J \Pi, U\rangle
$$

Therefore, the necessary condition associated with (3.11) is

$$
Q^{\top} P-\frac{1}{2} \Sigma(U)+\alpha J \Pi=0
$$

Since $U \in \mathfrak{g}$, and $\Sigma: \mathfrak{g} \rightarrow \mathfrak{g}$, the necessary condition (3.13) implies that $Q^{\top} P+$ $\alpha J \Pi \in \mathfrak{g}$. Therefore, we have

$$
J\left(Q^{\top} P+\alpha J \Pi\right)+\left(Q^{\top} P+\alpha J \Pi\right)^{\top} J=0 .
$$

Thus,

$$
\Pi=-\frac{1}{2}\left(J Q^{\top} P+P^{\top} Q J\right)
$$

Hence, (3.13) becomes

$$
Q^{\top} P-\frac{1}{2} \Sigma(U)-\frac{\alpha}{2} J\left(J Q^{\top} P+P^{\top} Q J\right)=0
$$

and therefore

$$
\Sigma(U)=Q^{\top} P-\alpha J P^{\top} Q J
$$


Using the above lemma, we see that $Q^{\top} P-\alpha J P^{\top} Q J \in \mathfrak{g}$. Thus, the optimal control for the control problem in Proposition 3.2 is given uniquely by

$$
U^{*}=2 \Sigma^{-1}\left(\mathrm{P}\left(Q^{\top} P\right)\right) \in \mathfrak{g}
$$

Since $U \in \mathfrak{g}$, it follows that the extremal flow (3.8) and (3.9) has an invariant set $G \times \mathfrak{g l}(n) \subset \mathfrak{g l}(n) \times \mathfrak{g l}(n)$. It follows that the flow restricted to the invariant set $G \times \mathfrak{g l}(n)$ captures all of the extremals of the original optimal control problem (3.2).

By Lemma 2.1, if $U \in \mathfrak{g}$, then $U^{\top} \in \mathfrak{g}$. So the system

$$
\frac{\mathrm{d}}{\mathrm{d} t} \bar{P}=-\bar{P} U^{\top}, \quad \bar{P}(0)=I
$$

evolves in the Lie group $G$. Now setting $P_{1}(t)=P_{0} \bar{P}(t)$, where $P_{0}=P(0)$ is a constant, we see that the time evolution of $P_{1}(t)$ and $P(t)$ are governed by the same equation (the second equation in (3.8)) and have the same initial condition $P_{1}(0)=$ $P(0)=P_{0}$. Therefore, $P_{1}(t) \equiv P(t)$ and $P(t)=P_{0} \bar{P}(t)$.

For $Q_{0} \in G$ and denoting the initial costate $P_{0}$ by $R$ enables us to conclude the following:

Proposition 3.4 All extremal trajectories for the problem (3.2) are realized from the system

$$
\begin{aligned}
& \dot{Q}=Q U, \quad Q(0)=Q_{0}, \quad Q \in G, \\
& \dot{\bar{P}}=-\bar{P} U^{\top}, \quad \bar{P}(0)=I, \quad \bar{P} \in G, \\
& U=2 \Sigma^{-1}\left(\mathrm{P}\left(Q^{\top} R \bar{P}\right)\right) \in \mathfrak{g},
\end{aligned}
$$

for suitable $R \in \mathfrak{g l}(n)$.

However, it is also clear that the optimal flow (3.8) and (3.9) has an invariant set $G \times G \subset \mathfrak{g l}(n) \times \mathfrak{g l}(n)$. In Proposition 3.4, this corresponds to the restriction $P(0)=$ $R$ to $G$. By restricting system (3.8) and (3.9) to $G \times G$, we may miss some of the extremal trajectories for problem (3.2) since we are restricting the costate trajectories $P$ to $G$. We elaborate on this statement at the end of Sect. 3.3 and in Sect. 4.

We now study the flow (3.8) and (3.9) restricted to $G \times G$. In this case, since $(Q, P) \in G \times G$, we have $Q^{\top} P \in G$ and $P^{\top} Q J=J\left(Q^{\top} P\right)^{-1}=\left(Q^{\top} P\right)^{\top} J$ so the expression in (3.9) simplifies to $U_{\text {ext }}=\Sigma^{-1}\left(Q^{\top} P-\left(Q^{\top} P\right)^{-1}\right)$. Notice that by Lemma 2.1 $Q^{\top} P-\left(Q^{\top} P\right)^{-1} \in \mathfrak{g}$ for all $(Q, P) \in G \times G$, so $U$ is well defined.

Because we have special interest in the extremal flow (3.8) and (3.9) restricted to $G \times G$, we write down the system

$$
\begin{aligned}
& \dot{Q}=Q U, \quad Q \in G, \\
& \dot{P}=-P U^{\top}, \quad P \in G, \\
& U=\Sigma^{-1} \mathrm{P}\left(Q^{\top} P\right)=\Sigma^{-1}\left(Q^{\top} P-\left(Q^{\top} P\right)^{-1}\right) \in \mathfrak{g} .
\end{aligned}
$$


Now the Hamiltonian $H$ in (3.7) may also be rewritten when it is restricted to $G \times G \times \mathfrak{g}$ since by Lemma 2.2

$$
\frac{1}{2}\left\langle Q^{\top} P, U\right\rangle=-\frac{1}{2}\left\langle\left(Q^{\top} P\right)^{-1}, U\right\rangle
$$

and so $H$ becomes

$$
H(P, Q, U)=\frac{1}{2}\left\langle Q^{\top} P-\left(Q^{\top} P\right)^{-1}, U\right\rangle-\frac{1}{4}\langle U, \Sigma(U)\rangle,
$$

and on inserting the form of $U$ in (3.19), we obtain

$$
H=\frac{1}{4}\left\langle Q^{\top} P-\left(Q^{\top} P\right)^{-1}, \Sigma^{-1}\left(Q^{\top} P-\left(Q^{\top} P\right)^{-1}\right)\right\rangle
$$

While the system (3.8) and (3.9) is a Hamiltonian flow on $\mathfrak{g l}(n) \times \mathfrak{g l}(n)$ with the canonical symplectic form, it is not obvious that the flow (3.18) and (3.19) on $G \times G$ is Hamiltonian. We now describe in what sense this restricted flow is Hamiltonian. We denote by $\Omega$ the restriction of $\Omega_{\text {can }}$ to $G \times G$. Further, we let $S_{\text {max }} \subset G \times G$ be the maximal open submanifold in $G \times G$ where $\Omega$ is nondegenerate. Clearly $S_{\max }$ is not empty as $\Omega=\Omega_{\text {can }}$ at the identity in $G \times G$.

The next result demonstrates that (3.18) and (3.19) restricted to the symplectic manifold $\left(S_{\max }, \Omega\right)$ is a Hamiltonian flow with the Hamiltonian given by (3.20).

Proposition 3.5 The restriction of the system (3.18) and (3.19) to $S_{\max } \in G \times G$ is Hamiltonian, with the Hamiltonian (3.20) and the flow described by (3.18) and (3.19).

Proof Let $X^{H}$ denote the Hamiltonian vector field of this Hamiltonian, and let $Z$ be another vector field on $G \times G$. Thus,

$$
\mathrm{d} H(Z)=\Omega\left(X^{H}, Z\right)=\left\langle X_{Q}^{H}, Z_{P}\right\rangle-\left\langle X_{P}^{H}, Z_{Q}\right\rangle .
$$

If we let $Z=\left(Z_{Q}, Z_{P}\right)=(Q V, P V)$, then

$$
\mathrm{d} H(Q V)=-\left\langle X_{P}^{H}, Q V\right\rangle, \quad d H(P V)=\left\langle X_{Q}^{H}, P V\right\rangle .
$$

Using (2.3), the first of these expressions can be expressed as

$$
\begin{aligned}
\mathrm{d} H(Q V) & =\frac{1}{2}\left\langle V^{\top} Q^{\top} P, U\right\rangle+\frac{1}{2}\left\langle P^{-1}\left(Q^{\top}\right)^{-1}(Q V)^{\top}\left(Q^{\top}\right)^{-1}, U\right\rangle \\
& =\frac{1}{2}\left\langle Q V, P U^{\top}\right\rangle+\frac{1}{2}\left\langle\left(Q^{\top}\right)^{-1} V^{\top},\left(P^{-1}\right)^{\top} U\right\rangle \\
& =\frac{1}{2}\left\langle Q V, P U^{\top}\right\rangle+\frac{1}{2} \operatorname{Trace}\left(Q^{-1}\left(P^{-1}\right)^{\top} U V\right) \\
& =\frac{1}{2}\left\langle Q V, P U^{\top}\right\rangle+\frac{1}{2} \operatorname{Trace}\left(P^{\top} Q V U\right) \quad \text { from }(2.3) \\
& =\frac{1}{2}\left\langle Q V, P U^{\top}\right\rangle+\frac{1}{2}\left\langle Q V, P U^{\top}\right\rangle=\left\langle Q V, P U^{\top}\right\rangle .
\end{aligned}
$$


Similarly, the second expression can also be simplified as follows

$$
\begin{aligned}
\mathrm{d} H(P V) & =\frac{1}{2}\left\langle Q^{\top} P V, U\right\rangle+\frac{1}{2}\left\langle P^{-1}(P V) P^{-1}\left(Q^{\top}\right)^{-1}, U\right\rangle \\
& =\frac{1}{2}\langle Q U, P V\rangle+\frac{1}{2}\left\langle V P^{-1}\left(Q^{\top}\right)^{-1}, U\right\rangle \\
& =\frac{1}{2}\langle Q U, P V\rangle+\frac{1}{2} \operatorname{Trace}\left(Q^{-1}\left(P^{-1}\right)^{\top} V^{\top} U\right) \\
& =\frac{1}{2}\langle Q U, P V\rangle+\frac{1}{2} \operatorname{Trace}\left(P^{\top} Q U V^{\top}\right) \quad \text { from }(2.3) \\
& =\frac{1}{2}\langle Q U, P V\rangle+\frac{1}{2}\langle Q U, P V\rangle=\langle Q U, P V\rangle .
\end{aligned}
$$

Hence, on the symplectic subset $S_{\max }$ of $G \times G$, we get $X_{P}^{H}=-P U^{\top}$ and $X_{Q}^{H}=Q U$.

\subsection{Extremal Flow in Terms of an Involution}

We now give another formulation of the extremal flow (3.18) and (3.19) for the optimal control problem, in which we eliminate explicit reference to the transpose operator. We introduce on GL $(n)$ (the Lie group of invertible $n \times n$ real matrices), the involution

$$
\sigma: \quad \mathrm{GL}(n) \rightarrow \mathrm{GL}(n) ; \quad \sigma(X)=\left(X^{\top}\right)^{-1} .
$$

Thus, $\sigma$ is an automorphism which satisfies $\sigma^{2}(\cdot)=I_{n}$, i.e., $\sigma$ is an involution on GL(n). Clearly, by Lemma 2.1, $\sigma$ restricts to an automorphism of $G$ as well.

The Lie group isomorphism $\sigma$ induces a Lie algebra automorphism of $\mathfrak{g}$ and $\mathfrak{g l}(n)$, given by

$$
\widehat{\sigma}: \quad \mathfrak{g} \rightarrow \mathfrak{g} ; \quad \widehat{\sigma}(A)=-A^{\top} .
$$

Hence, $\widehat{\sigma}^{2}(\cdot)=I_{n}$ is also an involution on $\mathfrak{g l}(n)$, and it is easy to directly check that $\mathfrak{g}$ is mapped to itself by the involution $\widehat{\sigma}$. If $A \in \mathfrak{g}$ and $X=\exp (A) \in G$ where $\exp : \mathfrak{g} \rightarrow G$ is the exponential map, then ${ }^{1}$

$$
\sigma(X)=\exp \left(-A^{\top}\right)=\exp (\widehat{\sigma}(A))
$$

Now if $\dot{Q}=Q U, Q \in G$ and $U \in \mathfrak{g}$, then

$$
\frac{\mathrm{d}}{\mathrm{d} t} \sigma(Q)=: \sigma_{*} \dot{Q}=\sigma(Q) \widehat{\sigma}(U)
$$

\footnotetext{
${ }^{1}$ According to [19], $\widehat{\sigma}$ is a Cartan involution, since the fixed point set of $\widehat{\sigma}$ is $\mathfrak{s o}(n)$, which is the Lie algebra of a maximal compact subgroup of $\operatorname{SL}(n)$, the adjoint group of $[\mathfrak{g l}(n), \mathfrak{g l}(n)]=\mathfrak{s l}(n)$.
} 
since $\dot{Q}^{\top}=U^{\top} Q^{\top}$ and $\left(Q^{\dot{\top}}\right)^{-1}=-\left(Q^{\top}\right)^{-1} U^{\top}$. Similarly

$$
\frac{\mathrm{d}}{\mathrm{d} t} \sigma(P)=\sigma_{\star} \dot{P}=\left(P^{\top}\right)^{-1} \dot{P}^{\top}\left(P^{\top}\right)^{-1}=\left(P^{\top}\right)^{-1} U=\sigma(P) U,
$$

where we used (3.18) in the last step. We define

$$
M \triangleq Q^{\top} P-\left(Q^{\top} P\right)^{-1}=Q^{\top} P-P^{-1}\left(Q^{\top}\right)^{-1}
$$

which gives

$$
M=\sigma\left(Q^{-1}\right) P-P^{-1} \sigma(Q)
$$

Therefore, we can also express the extremal flow (3.18) and (3.19) as

$$
\begin{aligned}
& \dot{Q}=Q U, \quad \dot{P}=P \widehat{\sigma}(U), \quad U=\Sigma^{-1}(M), \\
& M=\sigma\left(Q^{-1}\right) P-P^{-1} \sigma(Q) .
\end{aligned}
$$

We now use (3.25) to obtain the flow of $M$.

Lemma 3.6 The flow of the quantity $M$ along the extremal flow (3.25) is given by

$$
\dot{M}=[M, \widehat{\sigma}(U)]
$$

Proof We have to evaluate

$$
\dot{M}=\frac{\mathrm{d}}{\mathrm{d} t}\left(\sigma\left(Q^{-1}\right) P-P^{-1} \sigma(Q)\right) .
$$

Now $\sigma\left(Q^{-1}\right)=(\sigma(Q))^{-1}$, so using (3.23), we get

$$
\frac{\mathrm{d}}{\mathrm{d} t} \sigma\left(Q^{-1}\right)=-\widehat{\sigma}(U) \sigma\left(Q^{-1}\right)
$$

Hence, along the extremal flow (3.25) of the optimal control problem, we have

$$
\begin{aligned}
\dot{M} & =-\widehat{\sigma}(U) \sigma\left(Q^{-1}\right) P+\sigma\left(Q^{-1}\right) P \widehat{\sigma}(U)+\widehat{\sigma}(U) P^{-1} \sigma(Q)-P^{-1} \sigma(Q) \widehat{\sigma}(U) \\
& =\left[\sigma\left(Q^{-1}\right) P-P^{-1} \sigma(Q), \widehat{\sigma}(U)\right]=[M, \widehat{\sigma}(U)]
\end{aligned}
$$

which proves the lemma.

The following statement summarizes these results as a generalization of the $n$-dimensional rigid body (the Euler-Poincaré) equations.

Theorem 3.7 The "generalized Euler" equations for the optimal control problem (3.2) are given by

$$
\dot{Q}=Q U, \quad \dot{M}=[M, \widehat{\sigma}(U)], \quad U=\Sigma^{-1}(M) .
$$


Clearly the optimal control problem (3.2) can be expressed as a variational problem on $G$, which gives rise to geodesics on $G$ with a left invariant metric corresponding to $\Sigma$ on $\mathfrak{g}$. Reference [15] give a general form for the Euler-Poincaré equations for semisimple Lie groups (generalized rigid body) as a Hamiltonian flow on $T^{\star} G$. Equivalently, one could recast this as an optimal control problem on $G$ directly resulting in the same Hamiltonian flow on $T^{\star} G$, with the canonical symplectic form on $T^{\star} G$.

Now if $M$ satisfies the Lax equation in (3.27), then

$$
\dot{M}^{\top}=\left(M\left(-U^{\top}\right)-\left(-U^{\top}\right) M\right)^{\top}=M^{\top} U-U M^{\top}=\left[M^{\top}, U\right] .
$$

As in $[1,14]$, the Lax equations

$$
\dot{h}=[h, u], \quad u=\Sigma^{-1}(h), \quad u, h \in \mathfrak{g},
$$

represent the reduction of the geodesic flow on $T^{\star} G$ to the Poisson flow of the coadjoint equations on the dual of the Lie algebra. Our representation of these Lax equations follows since we have employed the trace inner product on $\mathfrak{g l}(n)$ with a transpose, whereas this is not the case in the texts mentioned above. Hence, the system (3.27) does recover the usual Euler-Poincaré equations for the geodesic flow on $G$. Note that in the case of $G=\operatorname{SO}(n)$, we have $\widehat{\sigma}(U)=U$ for $U \in \mathfrak{s o}(n)$ and either way we achieve the usual Euler-Poincaré equations for the $n$-dimensional rigid body.

Since the system (3.27) does capture all extremals for the optimal control problem (3.2), and we know that the system on $G \times G$ given by the flow (3.18) and (3.19) does not necessarily do so, the relationship between the two flows is not expected to be straightforward. To develop this relationship, we introduce the mapping

$$
\Phi: \quad G \times G \rightarrow G \times \mathfrak{g}, \quad(Q, P) \mapsto(Q, M)
$$

defined by $M=\sigma\left(Q^{-1}\right) P-P^{-1} \sigma(Q)$. Clearly by construction, the mapping $\Phi$ maps every trajectory of the flow (3.18) and (3.19) on $G \times G$ onto a trajectory of the flow of the generalized Euler equations (3.27). To explore the inverse of $\Phi$, we introduce the mapping $\sinh : \mathfrak{g} \rightarrow \mathfrak{g}$ defined by

$$
\sinh X=\frac{1}{2}(\exp (X)-\exp (-X)) .
$$

We note that $\sinh (\cdot)$ does indeed restrict to a map from $\mathfrak{g}$ to $\mathfrak{g}$ since if $X \in \mathfrak{g}$, $\exp (X) \in G$, and hence $\exp (X)-\exp (-X) \in \mathfrak{g}$, by Lemma 2.1. Now if we set

$$
P=\sigma(Q) \exp \left(\sinh ^{-1} \frac{M}{2}\right)
$$

in the expression for $M$, we obtain

$$
M=\exp \left(\sinh ^{-1} \frac{M}{2}\right)-\exp \left(-\sinh ^{-1} \frac{M}{2}\right) .
$$


Thus, as long as $\sinh ^{-1}$ is defined, the expression (3.29) does indeed define an inverse for $\Phi$. We investigate the domain of this inverse in the next section. In the case that $J=I_{n}, \alpha=1$ and $G=\mathrm{SO}(n),(3.25)$ and (3.27) reduce to the symmetric representation of the rigid body equations in $\mathrm{SO}(n)$ given in [3], where the map $\sinh : \mathfrak{s o}(n) \rightarrow \mathfrak{s o}(n)$ was first introduced.

\subsection{A Conserved Quantity Along the Extremal Flow}

From (3.18) or (3.25), we see that the quantity

$$
\gamma=P Q^{\top}=P \sigma(Q)^{-1}
$$

is conserved along the extremal flow (3.18) and (3.19) or (3.25), since

$$
\dot{\gamma}=\dot{P} Q^{\top}+P \dot{Q}^{\top}=-P U^{\top} Q^{\top}+P U^{\top} Q^{\top}=0
$$

We define the quantity

$$
m \triangleq \gamma-\gamma^{-1}=P Q^{\top}-\left(P Q^{\top}\right)^{-1}
$$

which is also conserved along the extremal flow (3.18) and (3.19) or (3.25). It follows that where $\sinh ^{-1}$ is defined, we obtain

$$
\gamma=\exp \left(\sinh ^{-1} \frac{m}{2}\right)
$$

and that from (3.30), we obtain

$$
P=\exp \left(\sinh ^{-1} \frac{m}{2}\right) \sigma(Q)=\gamma \sigma(Q) .
$$

It follows that expressions (3.31) and (3.29) are equivalent expressions for $P$, but the expression (3.31) is expressed in terms of the invariant $m$, as opposed to $M$ which is not invariant. Now let $g=Q^{\top} P$, so we can write

$$
\gamma=\left(Q^{\top}\right)^{-1}\left(Q^{\top} P\right) Q^{\top}=\sigma(Q) g \sigma(Q)^{-1},
$$

which implies that

$$
\gamma=\operatorname{Int}_{\sigma(Q)} g
$$

where for $s, g \in G$, the inner automorphism $\operatorname{Int}_{s}(g)=s g s^{-1}$ is an analytic isomorphism of $G$ onto itself (see [10]). If we denote the algebra elements

$$
a=\sinh ^{-1} \frac{m}{2} \quad \text { and } \quad A=\sinh ^{-1} \frac{M}{2},
$$

then (3.32) can also be expressed as

$$
\exp a=\operatorname{Int}_{\sigma(Q)} \exp A=\exp \left(\operatorname{Ad}_{\sigma(Q)} A\right)
$$


since the exponential map relates the inner automorphism on the group $G$ with the adjoint action of the group on its algebra $\mathfrak{g}$ (see $[10,18])$. This shows that

$$
a=\operatorname{Ad}_{\sigma(Q)} A \Rightarrow m=\operatorname{Ad}_{\sigma(Q)} M,
$$

which relates the quantities $m$ and $M$ through the adjoint action of the group $G$ on its algebra $\mathfrak{g}$ via the involution $\sigma$. As we show in the following section, the quantity $m$ is essential to defining an invariant open submanifold of $G \times G$ where the extremal flows given by (3.18) and (3.19) are Hamiltonian.

\section{Symplectic Submanifolds}

In this section, we exhibit domains on which the mapping $\Phi: G \times G \rightarrow G \times \mathfrak{g}$ has a well-defined inverse and on which $\Phi$ is naturally symplectic. We obtain a symplectic submanifold of $G \times G$ (that is, maximal in a norm-bounded sense) and show that it is symplectomorphic to $G \times g$ or $T^{\star} G$. Therefore, $\Phi$ maps the optimal trajectories given by the symmetric representation (3.18) and (3.19) in Proposition 3.5 in this submanifold to corresponding trajectories of the generalized Euler equations given by (3.27) in Theorem 3.27. We state the main result of this section below, but delay the proof until later in the section.

Theorem 4.1 The set $\left(S_{2}, \Omega\right)$ is a symplectic submanifold of $\left(\mathfrak{g l}(n) \times \mathfrak{g l}(n), \Omega_{\text {can }}\right)$ where

$$
S_{2} \triangleq\left\{(Q, P) \in G \times G \mid m=P \sigma\left(Q^{-1}\right)-\sigma(Q) P^{-1},\|m\|<2\right\},
$$

and $\|\cdot\|$ is the operator norm on $\mathfrak{g l}(n)$.

\subsection{Non-degeneracy Condition for the Canonical Symplectic Form}

The restriction of the canonical symplectic form $\Omega_{\text {can }}$ to $G \times G$ is given by

$$
\begin{aligned}
\Omega\left(\left(Q A_{1}, P B_{1}\right),\left(Q A_{2}, P B_{2}\right)\right) & =\left.\Omega_{\text {can }}\right|_{G \times G}\left(\left(Q A_{1}, P B_{1}\right),\left(Q A_{2}, P B_{2}\right)\right) \\
& =\left\langle P B_{2}, Q A_{1}\right\rangle-\left\langle P B_{1}, Q A_{2}\right\rangle \\
& =\operatorname{Trace}\left(B_{2}^{\top} P^{\top} Q A_{1}-B_{1}^{\top} P^{\top} Q A_{2}\right),
\end{aligned}
$$

for $(Q, P) \in G \times G$ and $\left(A_{1}, B_{1}\right),\left(A_{2}, B_{2}\right) \in \mathfrak{g} \times \mathfrak{g}$.

Let $S_{\max }$ be the maximal submanifold (with respect to inclusion) of $G \times G$ on which $\Omega_{\text {can }}$ restricts to a nondegenerate symplectic form $\Omega$. For a given point $(Q, P) \in S_{\max }$, nondegeneracy of $\Omega$ at $(Q, P)$ is equivalent to the condition: if

$$
\operatorname{Trace}\left(B_{2}^{\top} P^{\top} Q A_{1}-B_{1}^{\top} P^{\top} Q A_{2}\right)=0
$$

for all $\left(A_{2}, B_{2}\right) \in \mathfrak{g} \times \mathfrak{g}$, then $\left(A_{1}, B_{1}\right)=(0,0)$. This is equivalent to requiring that for $A \in \mathfrak{g}$,

$$
\operatorname{Trace}\left(B^{\top} P^{\top} Q A\right)=0
$$


for all $B \in \mathfrak{g}$ implies that $A=0$. Using the projection P in Lemma 3.3, we know that for $A \in \mathfrak{g l}(n)$,

$$
A=\mathrm{P}(A)+(\mathrm{I}-\mathrm{P})(\mathrm{A})
$$

where

$$
\mathrm{P}(A)=\frac{1}{2}\left(A-\alpha J A^{\top} J\right), \quad(\mathrm{I}-\mathrm{P})(A)=\frac{1}{2}\left(A+\alpha J A^{\top} J\right) .
$$

Moreover, since $\mathrm{P}=\mathrm{P}^{\star}$, we have

$$
\langle(\mathrm{I}-\mathrm{P})(A), \mathrm{P}(B)\rangle=\langle J(\mathrm{I}-\mathrm{P})(A), J \mathrm{P}(B)\rangle=\left\langle\left(\mathrm{P}-\mathrm{P}^{2}\right)(A), B\right\rangle=0 .
$$

Thus,

$$
\mathfrak{g l}(n)=J \mathrm{P}(\mathfrak{g l}(n)) \oplus J(\mathrm{I}-\mathrm{P})(\mathfrak{g l}(\mathrm{n})) .
$$

is an orthogonal decomposition. Note that

$$
\begin{aligned}
(J \mathrm{P}(A))^{\top} & =\frac{1}{2} \alpha\left(A-\alpha J A^{\top} J\right)^{\top} J=\frac{1}{2} \alpha\left(A^{\top}-\alpha J A J\right) J \\
& =\frac{1}{2} J\left(J A^{\top} J-\alpha A\right)=-\frac{1}{2} \alpha J\left(A-\alpha J A^{\top} J\right)=-\alpha J \mathrm{P}(A)
\end{aligned}
$$

and similarly

$$
\begin{aligned}
(J(\mathrm{I}-\mathrm{P})(\mathrm{A}))^{\top} & =\frac{1}{2} \alpha\left(A+\alpha J A^{\top} J\right)^{\top} J=\frac{1}{2} \alpha\left(A^{\top}+\alpha J A J\right) J \\
& =\frac{1}{2} J\left(J A^{\top} J+\alpha A\right)=\frac{1}{2} \alpha J\left(A+\alpha J A^{\top} J\right)=\alpha J(\mathrm{I}-\mathrm{P})(\mathrm{A}) .
\end{aligned}
$$

Let $g=Q^{\top} P \in G$ so that

$$
\operatorname{Trace}\left(B^{\top} P^{\top} Q A\right)=\operatorname{Trace}\left(B^{\top} g^{\top} A\right)=\operatorname{Trace}\left((J B)^{\top} J g^{\top} A\right)=\left\langle J B, J g^{\top} A\right\rangle \text {. }
$$

Now $J B \in J \mathrm{P}(\mathfrak{g l}(n))$ for all $B \in \mathfrak{g}$, and so $\left\langle J B, J g^{\top} A\right\rangle=0$ for all $B \in \mathfrak{g}$ implies by (4.3) that $J g^{\top} A \in J(\mathrm{I}-\mathrm{P})(\mathfrak{g l}(\mathrm{n}))$. Thus, from the above

$$
\left(J g^{\top} A\right)^{\top}=\alpha J g^{\top} A \Rightarrow A^{\top} g J=J g^{\top} A \Rightarrow-J A J^{\top} g J=J g^{\top} A
$$

However, $g^{\top} J g=J \Rightarrow\left(g^{\top}\right)^{-1}=J^{\top} g J$ so $-A\left(g^{\top}\right)^{-1}=g^{\top} A$, or simply

$$
\sigma(g) A \sigma(g)=-A, A \in \mathfrak{g} .
$$

We conclude that if $(Q, P) \in S_{\max }$ and $g=Q^{\top} P$, then (4.4) must imply that $A=0$.

We, therefore, have the following characterization of $S_{\max }$, the maximal symplectic submanifold of $G \times G$ with the symplectic form induced from $\Omega_{\text {can }}$ :

$$
S_{\max }=\left\{(Q, P) \in G \times G: g=Q^{\top} P, \sigma(g) A \sigma(g)=-A \text { for } A \in \mathfrak{g} \Rightarrow A=0\right\} .
$$

We next look at the condition (4.4) in more detail to obtain a symplectic submanifold of $G \times G$ with a computable boundary. 


\subsection{A Symplectic Submanifold of $G \times G$}

To begin with, we give some basic results related to the matrix functions exp and sinh. Let the matrices $\Psi$ and $V$ be the matrices of eigenvalues and eigenvectors, respectively, of $C$; i.e., $C V=V \Psi$. Then we have:

$$
\begin{aligned}
\exp (C) V & =\left\{I+C+\frac{1}{2} C^{2}+\frac{1}{3 !} C^{3}+\cdots\right\} V \\
& =V+C V+\frac{1}{2} C^{2} V+\frac{1}{3 !} C^{3} V+\cdots \\
& =V+V \Psi+\frac{1}{2} V \Psi^{2}+\frac{1}{3 !} V \Psi^{3}+\cdots \\
& =V\left\{I+\Psi+\frac{1}{2} \Psi^{2}+\frac{1}{3 !} \Psi^{3}+\cdots\right\}=V D,
\end{aligned}
$$

where $D=\exp (\Psi)$. Similarly, it can be shown that

$$
\exp (-C) V=V \exp (-\Psi)
$$

Combining these two results, we obtain:

$$
\begin{aligned}
(\sinh C) V & =\frac{1}{2}(\exp (C)-\exp (-C)) V \\
& =\frac{1}{2} V(\exp (\Psi)-\exp (-\Psi))=V \sinh \Psi .
\end{aligned}
$$

If $B=\sinh C$, then this means that $B V=V \Lambda$, where $\Lambda=\sinh \Psi$. We use the operator norm $\|\cdot\|$ on $\mathfrak{g l}(n)$ defined by

$$
\|A\|=\sup \{\|A x\| \mid\|x\|=1\} .
$$

We know that the function sinh $: \mathbb{C} \rightarrow \mathbb{C}$ has an inverse $\sinh ^{-1}(u)=\ln \left(u+\sqrt{u^{2}+1}\right)$ with a convergent power series expansion for $|u|<1$ (see [11]). This power series expansion can be used to show that the map $\sinh : \mathfrak{g} \rightarrow \mathfrak{g}$ has an inverse on the set $U=\{A \in \mathfrak{g} \mid\|A\|<1\}$, and we denote this inverse $\sinh ^{-1}: U \rightarrow \mathfrak{g}$ as in [3]. The following lemma gives a sufficient condition under which condition (4.4) implies that $A=0$ where $A \in \mathfrak{g}$.

Lemma 4.2 For $A, B \in \mathfrak{g}$, if $\|B\|<1$ and

$$
\exp \left(\sinh ^{-1} B\right) A \exp \left(\sinh ^{-1} B\right)=-A,
$$

then $A=0$.

Proof Note that we do not make any assumptions on the diagonalizability of $B \in \mathfrak{g}$. Let $\left\{v_{1}, v_{2}, \ldots, v_{m}\right\}, m \leq n$, be the set of independent eigenvectors of $B$, and let 
$\lambda_{k} \in \mathbb{C}$ denote the eigenvalue of $B$ corresponding to the eigenvector $v_{k} \in \mathbb{C}^{n}$. Then we have

$$
\left(\sinh ^{-1} B\right) v_{k}=\left(\sinh ^{-1} \lambda_{k}\right) v_{k}, \quad k=1, \ldots, m .
$$

If we denote $g=\exp \left(\sinh ^{-1} B\right)$, then from the above equation, we get

$$
g v_{k}=d_{k} v_{k}, \quad k=1, \ldots, m,
$$

where $d_{k}=\exp \left(\sinh ^{-1} \lambda_{k}\right) \in \mathbb{C}$ is the eigenvalue of $g$ corresponding to the eigenvector $v_{k}$. Now right-multiplication of (4.5) with $v_{k}$ gives $g A g v_{k}=-A v_{k}$; that is, $g A\left(d_{k} v_{k}\right)=-A v_{k}$, i.e., $d_{k}\left(g f_{k}\right)=-f_{k}$. In other words,

$$
g^{-1} f_{k}=-d_{k} f_{k}
$$

where $f_{k}=A v_{k}$.

Hence, the vector $f_{k}=A v_{k}$ and the scalar quantity $-d_{k}$ form an eigenvectoreigenvalue pair for $g^{-1}=\exp \left(-\sinh ^{-1} B\right)$. But since $g \in G$ is a nonsingular matrix, $g^{-1} v_{l}=\frac{1}{d_{l}} v_{l}$, i.e., the eigenvalues of $g^{-1}$ are also given by the reciprocals of the $d_{l}$. Equation (4.6) is trivially satisfied if $A=0$, since in that case, $f_{k}=A v_{k}=0$. For $A \neq 0$, we have at least one nonzero eigenvector-eigenvalue pair. Since the number of independent eigenvectors and the corresponding eigenvalues of $g^{-1}$ are unique, (4.6) is equivalent to the presence of at least one pair $(k, l) \in\{1, \ldots, m\}$ such that $\left(1+d_{k} d_{l}\right)=0$. Now if $\lambda_{k}=r_{k} \exp \left(i \theta_{k}\right)$ are the eigenvalues of $B$, then the corresponding eigenvalues of $C=\sinh ^{-1} B$ are

$\sinh ^{-1} \lambda_{k}=\alpha_{k}+i \beta_{k}, \quad$ where $r_{k} \cos \theta_{k}=\sinh \alpha_{k} \cos \beta_{k}, r_{k} \sin \theta_{k}=\cosh \alpha_{k} \sin \beta_{k}$,

and $d_{k}=\left(\exp \alpha_{k}\right)\left(\cos \beta_{k}+i \sin \beta_{k}\right)$ are the eigenvalues of $g$. Thus, we have

$$
\begin{aligned}
r_{k}^{2} & =\sinh ^{2} \alpha_{k} \cos ^{2} \beta_{k}+\cosh ^{2} \alpha_{k} \sin ^{2} \beta_{k} \\
& =\sinh ^{2} \alpha_{k} \cos ^{2} \beta_{k}+\left(1+\sinh ^{2} \alpha_{k}\right) \sin ^{2} \beta_{k}=\sinh ^{2} \alpha_{k}+\sin ^{2} \beta_{k} .
\end{aligned}
$$

We know that $\|B\|$ is the square root of the maximum eigenvalue of $B^{\top} B$, which is also the largest singular value of $B$; we also know that the largest absolute eigenvalue is less than or equal to the largest singular value [9]. Hence, if $\|B\|<1$, then the eigenvalues of $B$ satisfy $\left|\lambda_{k}\right|=r_{k}<1$ for all $k \in\{1, \ldots, n\}$. Thus, from the previous expression for $r_{k}^{2}$, we have

$$
\sin ^{2} \beta_{k}=r_{k}^{2}-\sinh ^{2} \alpha_{k}<1-\sinh ^{2} \alpha_{k}<1 \Rightarrow\left|\sin \beta_{k}\right|<1,
$$

which ensures that the principal value of $\beta_{k} \in\left(-\frac{\pi}{2}, \frac{\pi}{2}\right)$. It is easy to verify that if $\left|\beta_{k}\right|<\frac{\pi}{2}$ for all $k \in\{1, \ldots, n\}$, then

$$
d_{k} d_{l}=\exp \left(\alpha_{k}+\alpha_{l}\right) \exp \left(i\left(\beta_{k}+\beta_{l}\right)\right)
$$

is never a negative real number, and thus $1+d_{k} d_{l} \neq 0$ for any pair $(k, l) \in\{1, \ldots, n\}$. This implies that if (4.6) (alternatively (4.5)) is satisfied, then we must have $F=0$, and thus $A=0$. This completes the proof. 
Define the subset $\breve{S} \subset G \times G$ by

$$
\breve{S} \triangleq\left\{(Q, P) \in G \times G \mid M=\sigma\left(Q^{-1}\right) P-P^{-1} \sigma(Q),\left\|\frac{\widehat{\sigma}(M)}{2}\right\|<1 \Rightarrow\|M\|<2\right\} .
$$

Note that by (3.29), if $\|M\|<2$, then

$$
P=\sigma(Q) \exp \left(\sinh ^{-1} \frac{M}{2}\right)
$$

is well defined, and with $g=\exp \left(\sinh ^{-1} \frac{M}{2}\right)$ so $\sigma(g)=\exp \left(\sinh ^{-1} \frac{\widehat{\sigma}(M)}{2}\right)$ and $\left\|\frac{\widehat{\sigma}(M)}{2}\right\|<1$. Thus, by Lemma 4.2 and (4.4) we deduce that on the set $\breve{S}, \Omega$ is nondegenerate, and hence $(\Omega, \breve{S})$ is a symplectic submanifold. Unfortunately, the dynamics of $M$ given by (3.27) does not preserve $\|M\|$ in general since it evolves by conjugation by elements of $G$, and $G$ may not be $\mathrm{SO}(n)$. Thus, $\breve{S}$ is not an invariant set for the flow (3.25) or (3.18) and (3.19).

This difficulty is overcome by expressing the nondegeneracy condition (4.4), in terms of the conserved quantity $m$, rather than $M$. To do this, we will utilize some of the relations developed in Sect. 3.4. From relation (3.32), we have

$$
g=\sigma(Q)^{-1} \gamma \sigma(Q)=\operatorname{Int}_{\sigma(Q)^{-1}} \gamma
$$

which yields

$$
\sigma(g)=Q^{-1} \sigma(\gamma) Q=\operatorname{Int}_{Q^{-1}} \sigma(\gamma) .
$$

Now we rewrite the nondegeneracy condition (4.4) replacing $g$ with $\gamma$, as follows:

$$
\text { Int }_{Q^{-1}} \sigma(\gamma) A \text { Int }_{Q^{-1}} \sigma(\gamma)=-A,
$$

which gives

$$
Q^{-1} \sigma(\gamma) Q A Q^{-1} \sigma(\gamma) Q=-A
$$

that is,

$$
\sigma(\gamma) \operatorname{Ad}_{Q} A \sigma(\gamma)=-\operatorname{Ad}_{Q} A .
$$

Now we prove the main result of this section, Theorem 4.1 .

Proof Note first that if $\|m\|<2$, then $\gamma=\exp \left(\sinh ^{-1} \frac{m}{2}\right)$ is well defined and $\left\|\frac{\widehat{\sigma}(m)}{2}\right\|<1$, so

$$
\sigma(\gamma)=\exp \left(\sinh ^{-1} \frac{\widehat{\sigma}(m)}{2}\right)
$$

is also well defined. Thus, if $(Q, P) \in S_{2}$ we may apply Lemma 4.2 to (4.8) and deduce that $\operatorname{Ad}_{Q} A=0$. However, since $Q \in G$, we deduce that $A=0$ and so $\Omega$ is nondegenerate at $(Q, P)$, and we conclude that $\Omega$ is nondegenerate on all of $S_{2}$.

We now show that if $G=\operatorname{Sp}(2 n)$, then the norm bound giving the symplectic submanifold $S_{2} \subset G \times G$ in Theorem 4.1 is indeed a tight bound. To show this, we 
investigate the condition $g B g=-B$ where $g \in \operatorname{Sp}(2 n)$ and $B \in \mathfrak{s p}(2 n)$, and find an example where this condition is satisfied for $B \neq 0$. Now if

$$
J=\left[\begin{array}{cc}
0 & I_{n} \\
-I_{n} & 0
\end{array}\right], \quad B=\left[\begin{array}{cc}
X & Y \\
Z & W
\end{array}\right] \in \mathfrak{s p}(2 n),
$$

then $Z=Z^{\top}, Y=Y^{\top}$, and $W=-X^{\top}$, since $B^{\top} J+J B=0$. If

$$
g=\left[\begin{array}{cc}
A & B \\
C & D
\end{array}\right] \in \mathrm{Sp}(2 n),
$$

then $A^{\top} C=C^{\top} A, B^{\top} D=D^{\top} B$, and $A^{\top} D-C^{\top} B=I_{n}$. Take $g=J$, then $A=D=$ $0, B=I_{n}, C=-I_{n}$. Now the condition $g B g=-B$ requires

$$
\left[\begin{array}{cc}
-W & Z \\
Y & -X
\end{array}\right]=-\left[\begin{array}{cc}
X & Y \\
Z & W
\end{array}\right]
$$

so $W=X$ and $Z=-Y$. Also since $B \in \mathfrak{s p}(2 n)$ we have that $B=\left[\begin{array}{cc}X & Y \\ -Y & X\end{array}\right]$ where $X=-X^{\top}, Y=Y^{\top}$. This indeed gives us a nonzero $B \in \mathfrak{s p}(2 n)$ such that $g B=-B$ for $g=J \in \operatorname{Sp}(2 n)$.

We would like to express $g=\exp \left(\sinh ^{-1} \frac{m}{2}\right)$ for $m \in \mathfrak{s p}(2 n)$. Note that $\exp (\alpha J)=I_{2 n} \cos \alpha+J \sin \alpha$. Thus, $\exp \left(\frac{\pi}{2} J\right)=J$ and $\exp \left(-\frac{\pi}{2} J\right)=-J$. Hence, we have

$$
\sinh \left(\frac{\pi}{2} J\right)=\frac{1}{2}(J-(-J))=J,
$$

and, therefore, we write $\frac{\pi}{2} J=\sinh ^{-1} J$ noting that $\sinh ^{-1}$ is multi-valued outside $U \subset \mathfrak{s p}(2 n)$, where $\sinh ^{-1}$ is uniquely defined. Note also that $J \in \operatorname{Sp}(2 n) \cap \mathfrak{s p}(2 n)$, and so $J=\exp \left(\sinh ^{-1} J\right)$. Therefore, we may identify $m=2 J \in \mathfrak{s p}(2 n)$ and $J=$ $\exp \left(\sinh ^{-1} \frac{m}{2}\right) \in \operatorname{Sp}(2 n)$. The condition in Lemma 4.2 states that if $g B g=-g$ where $g=\exp \left(\sinh ^{-1} \frac{m}{2}\right)$ and $\|m / 2\|<1$, then $B=0$. In this case, $m=2 J$ and clearly $\|m / 2\|=1$, since the spectral radius of $J$ is 1 as all eigenvalues are $\pm i$. Thus, our choice of $g=J$ is not included in the sufficient condition of Theorem 4.1 as $g \notin S_{2}$. Therefore, we conclude that the norm bound provided in Theorem 4.1 for the symplectic submanifold $S_{2} \subset G \times G$ is tight.

\subsection{Symplectic Nature of the Map from $G \times G$ to $G \times \mathfrak{g}$}

Recall the map $\Phi: G \times G \rightarrow G \times \mathfrak{g}$ given by (3.28). We may identify $G \times \mathfrak{g}$ with the cotangent bundle $T^{\star} G$ by the map

$$
\iota: \quad G \times \mathfrak{g} \rightarrow T^{\star} G ; \quad \imath(Q, M)=\left(Q,\left\langle\left(Q^{\top}\right)^{-1} M, \cdot\right\rangle\right) .
$$

If $\omega$ denotes the natural symplectic form on $T^{\star} G$ then its pullback $l^{\star} \omega$ is a symplectic form on $G \times \mathfrak{g}$. The symplectic form $l^{\star} \omega$ is easily described, as in [14], for example, as

$$
\left(l^{\star} \omega\right)_{(Q, M)}\left(\left(Q U_{1}, N_{1}\right),\left(Q U_{2}, N_{2}\right)\right)=\left\langle N_{2}, U_{1}\right\rangle-\left\langle N_{1}, U_{2}\right\rangle+\left\langle M,\left[U_{1}, U_{2}\right]\right\rangle,
$$


where $\left(U_{1}, U_{2}\right),\left(N_{1}, N_{2}\right) \in \mathfrak{g} \times \mathfrak{g}$. Set

$$
S_{M} \triangleq\left\{(Q, M) \in \mathfrak{g} \times \mathfrak{g} \mid\|m\|<2, m=\operatorname{Ad}_{\sigma(Q)} M\right\} .
$$

Then clearly $\Phi$ maps $S_{2}$ onto $S_{M}$. Also, $S_{2}$ is an invariant set for the system (3.27). Thus, $\Phi$ maps trajectories of the extremal flow (3.18) and (3.19) in $S_{2}$ onto trajectories of (3.27) in $S_{M}$. Moreover, on $S_{M}, \Phi$ has an unique inverse map

$$
\begin{aligned}
& \Phi^{-1}: S_{M} \subset G \times \mathfrak{g} \rightarrow S_{2} \subset G \times G \\
& (Q, M) \rightarrow(Q, P) ; \quad P=\sigma(Q) \exp \left(\sinh ^{-1} \frac{M}{2}\right) .
\end{aligned}
$$

We conclude this section by demonstrating that $\Phi$ is indeed a symplectic map from $S_{2}$ to $S_{M}$.

Theorem 4.3 The map $\Phi: S_{2} \subset G \times G \rightarrow S_{M} \subset G \times \mathfrak{g}$ is a symplectomorphism of $\left(\Omega, S_{2}\right)$ onto $\left(l^{\star} \omega, S_{M}\right)$ with $\Phi^{\star}\left(l^{\star} \omega\right)=2 \Omega$.

Proof Let $Z_{1}=\left(Q U_{1},-P V_{1}^{\top}\right)$ and $Z_{2}=\left(Q U_{2},-P V_{2}^{\top}\right)$ be two vectors in $T_{Q} G \times$ $T_{P} G$. We need to show that

$$
\left(\iota^{\star} \omega\right)\left(\Phi_{\star} Z_{1}, \Phi_{\star} Z_{2}\right)=2 \Omega\left(Z_{1}, Z_{2}\right)
$$

From (3.5), we see that

$$
\begin{aligned}
\Omega\left(Z_{1}, Z_{2}\right) & =\left\langle-P V_{2}^{\top}, Q U_{1}\right\rangle-\left\langle-P V_{1}^{\top}, Q U_{2}\right\rangle \\
& =-\left\langle Q^{\top} P, U_{1} V_{2}\right\rangle+\left\langle Q^{\top} P, U_{2} V_{1}\right\rangle .
\end{aligned}
$$

By Corollary 2.3, we find that

$$
\Omega\left(Z_{1}, Z_{2}\right)=\frac{1}{2}\left\langle Q^{\top} P, U_{2} V_{1}-U_{1} V_{2}\right\rangle+\frac{1}{2}\left\langle\left(Q^{\top} P\right)^{-1}, V_{1} U_{2}-V_{2} U_{1}\right\rangle .
$$

Let $N=\dot{M}=\frac{\mathrm{d}}{\mathrm{d} t}\left(Q^{\top} P-\left(Q^{\top} P\right)^{-1}\right)$, and computing along (3.18)

$$
\frac{\mathrm{d}}{\mathrm{d} t}\left(Q^{\top} P\right)^{-1}=V^{\top}\left(Q^{\top} P\right)^{-1}-\left(Q^{\top} P\right)^{-1} U^{\top}
$$

Thus, $\Phi_{\star} Z_{k}=\left(Q U_{k}, N_{k}\right)$ for $k=1,2$, where

$$
N_{k}=U_{k}^{\top} Q^{\top} P-Q^{\top} P V_{k}^{\top}-\left(V_{k}^{\top}\left(Q^{\top} P\right)^{-1}-\left(Q^{\top} P\right)^{-1} U_{k}^{\top}\right) .
$$

Thus, from (4.9),

$$
\left(l^{\star} \omega\right)\left(\Phi_{\star} Z_{1}, \Phi_{\star} Z_{2}\right)=\left\langle U_{1}, N_{2}\right\rangle-\left\langle U_{2}, N_{1}\right\rangle+\left\langle M,\left[U_{1}, U_{2}\right]\right\rangle
$$

where $M=Q^{\top} P-\left(Q^{\top} P\right)^{-1}$. One concludes from $(4.11)$ that $\left(l^{\star} \omega\right)\left(\Phi_{\star} Z_{1}, \Phi_{\star} Z_{2}\right)=$ $2 \Omega\left(Z_{1}, Z_{2}\right)$. 
We can now give a complete answer to the question we posed earlier in Sect. 3.2; whether or not the extremal flows (3.8) and (3.9) of problem (3.6) restricted to $G \times G$, namely the extremal flows (3.18) and (3.19), capture all or some optimal trajectories. We know that the generalized Euler flow on $G \times \mathfrak{g}$ given by (3.27) does capture all optimal trajectories. The nature of the mapping $\Phi: S_{2} \subset G \times G \rightarrow S_{M} \subset G \times \mathfrak{g}$ demonstrates that only the extremal flows evolving on $S_{M}$, or equivalently with $\|m\|<2$, are manifested within the dynamics (3.18) and (3.19). In the case $G=\mathrm{SO}(n)$, the condition $\|m\|<2$ is equivalent to $\|M\|<2$, and hence directly limits the magnitude of "velocity" $U=\Sigma^{-1}(M)$ in the kinematic equation $\dot{Q}=Q U$. For fixed $Q_{0} \in G$, the geodesic neighborhood of final states $Q(T)$, corresponding to each choice of $m$, $\|m\|<k$, can be made arbitrarily small by choosing $T$ suitably small. Denote this set by $\mathcal{R}\left(T, Q_{0}, k\right) \subset G$. If we now try to solve the optimal control problem (3.2) when $Q_{T} \notin \mathcal{R}\left(T, Q_{0}, 2\right)$, it is clear that the optimal trajectory must correspond to an extremal trajectory which does not satisfy $\|m\|<2$. Thus, the extremal flow (3.18) and (3.19) on $S_{2} \subset G \times G$ cannot capture this optimal trajectory. However, this can be circumvented through Proposition 3.4, in which the control function is modified or rescaled.

\section{The Discrete Optimal Control Problem}

In this section, we briefly treat the discrete version of the continuous optimal control problem (3.2). We first define the discrete optimal control problem on the quadratic group $G$ and then provide its solution in terms of the optimal trajectories and the optimal controls.

Let $\Lambda$ be a matrix satisfying the conditions of Proposition 3.1, i.e., $\Lambda$ satisfies $\Lambda^{\top} J=J \Lambda$ and $\Lambda+\Lambda^{\top}$ is positive definite. Let $\bar{Q}_{0}, \bar{Q}_{N} \in G$ be given fixed endpoints. We define the optimal control problem

$$
\min _{U_{k}} \sum_{k=1}^{N}\left\langle\Delta, U_{k}\right\rangle, \quad \Delta=\frac{1}{2}\left(\Lambda+\Lambda^{\top}\right),
$$

subject to the dynamics and initial and final data

$$
Q_{k+1}=Q_{k} U_{k}, \quad Q_{0}=\bar{Q}_{0}, Q_{N}=\bar{Q}_{N}
$$

for $Q_{k}, U_{k} \in G$. Note that we may restrict ourselves here to $U_{k}$ in a neighborhood of the identity element in the group $G$ if we take the iteration procedure to be such that steps are small, giving rise to small increments in $Q_{k}$.

\subsection{The Discrete Hamiltonian}

The discrete Hamiltonian corresponding to the discrete optimal control problem (5.1) subject to the constraints (5.2) is given by

$$
H\left(P_{k+1}, Q_{k}, U_{k}\right)=\left\langle P_{k+1}, Q_{k} U_{k}\right\rangle-\left\langle\Delta, U_{k}\right\rangle=\left\langle Q_{k}^{\top} P_{k+1}-\Delta, U_{k}\right\rangle,
$$


where $P_{k+1} \in \mathfrak{g l}(n)$ is the costate variable used to impose the kinematic constraint (5.2). Note that this Hamiltonian functional is linear in the controls, unlike the Hamiltonian (3.7) for the continuous optimal control problem. Before we obtain the main result giving discrete extremal trajectories corresponding to this Hamiltonian, we give a lemma below that is used to obtain the main result.

Lemma 5.1 The map $\Sigma_{D}: G \rightarrow \mathfrak{g}$ defined by

$$
\Sigma_{D}\left(U_{k}\right) \triangleq g \Delta-\Delta g^{-1}, \quad g \in G,
$$

is a diffeomorphism from a neighborhood of the identity in $G$ to a neighborhood of $0 \in \mathfrak{g}$.

Proof Note that the derivative of $\Sigma_{D}$ at the identity along

$$
\dot{g}=g U
$$

is $\Sigma: \mathfrak{g} \rightarrow \mathfrak{g}$ defined earlier by (3.4). Since $\Sigma$ is an invertible map, $\Sigma_{D}$ is, therefore, a diffeomorphism from a neighborhood of the identity in $G$ to a neighborhood of 0 in $\mathfrak{g}$.

Therefore, $\Sigma_{D}: G \rightarrow \mathfrak{g}$ is in this sense the discrete version of the continuous map $\Sigma: \mathfrak{g} \rightarrow \mathfrak{g}$. The inverse of the map $\Sigma_{D}$, when it exists, is given in Sect. 5.2. The following result gives the discrete extremal trajectories corresponding to the Hamiltonian (5.3).

Theorem 5.2 A solution of the discrete optimal control problem (5.1) is given by a sequence of matrices $\left(Q_{k}, P_{k}\right)$ in $G \times \mathfrak{g l}(n)$ satisfying the optimal evolution equations

$$
Q_{k+1}=Q_{k} U_{k}, \quad P_{k+1}=P_{k} \sigma\left(U_{k}\right),
$$

where $\sigma: \mathrm{GL}(n) \rightarrow \mathrm{GL}(n)$ is the involution defined by (3.21), and $U_{k}$ is given by

$$
U_{k}=\Sigma_{D}^{-1}\left(P_{k}^{\top} Q_{k}-\left(P_{k}^{\top} Q_{k}\right)^{-1}\right),
$$

when $U_{k}$ is in the neighborhood of the identity in $G$ where $\Sigma_{D}^{-1}$ exists.

Proof From the discrete Hamiltonian (5.3), we get

$$
P_{k}=\nabla_{Q_{k}} H=\left(U_{k} P_{k+1}^{\top}\right)^{\top}=P_{k+1} U_{k}^{\top},
$$

from which we obtain the discrete evolution (5.5). Note that as in the continuous case, the discrete extremal flow can be naturally restricted to $G \times G$ since if $P_{k} \in G$, then (5.7) implies that $P_{k+1} \in G$.

We now need to find the critical points of $H\left(P_{k+1}, Q_{k}, U_{k}\right)$ where $U_{k}^{\top} J U_{k}=J$ since $U_{k} \in G$. This Hamiltonian is of the form $\left\langle A_{k}, U_{k}\right\rangle$ where $A_{k}=Q_{k}^{\top} P_{k+1}-\Delta$. Applying Pontryagin's principle, we maximize the functional

$$
H_{a}\left(P_{k+1}, Q_{k}, U_{k}\right)=\left\langle A_{k}, U_{k}\right\rangle+\frac{1}{2}\left\langle\Pi, U_{k}^{\top} J U_{k}-J\right\rangle,
$$


with respect to $U_{k}$, where $\Pi$ is a Lagrange multiplier for the constraint $U_{k}^{\top} J U_{k}=J$. Since $\left(U_{k}^{\top} J U_{k}-J\right)^{\top}=\alpha\left(U_{k}^{\top} J U_{k}-J\right)$, we require that $\Pi^{\top}=\alpha \Pi$. We set the differential with respect to $U_{k}$ to be zero:

$$
\delta_{U_{k}} H_{a}=\left\langle A_{k}, \delta U_{k}\right\rangle+\left\langle\alpha J U_{k} \Pi, \delta U_{k}\right\rangle=0 .
$$

Therefore, the gradient $\nabla_{U_{k}} H_{a}=0$ when

$$
A_{k}+\alpha J U_{k} \Pi=Q_{k}^{\top} P_{k+1}-\Delta+\alpha J U_{k} \Pi=0,
$$

from which we get $\Pi=-U_{k}^{-1} J A_{k}$. Utilizing the property that $\Pi^{\top}=\alpha \Pi$, we get:

$$
\begin{aligned}
& \left(Q_{k}^{\top} P_{k+1}-\Delta\right)^{\top} J \sigma\left(U_{k}\right)=U_{k}^{-1} J\left(Q_{k}^{\top} P_{k+1}-\Delta\right) \\
& \quad \Rightarrow \quad\left(P_{k+1}^{\top} Q_{k}-\Delta\right) U_{k} J=J U_{k}^{\top}\left(Q_{k}^{\top} P_{k+1}-\Delta\right) .
\end{aligned}
$$

Equivalently, making the substitutions $U_{k} \rightarrow U_{k}^{\top}, P_{k+1}^{\top} Q_{k} \rightarrow Q_{k}^{\top} P_{k+1}$, we get:

$$
\begin{aligned}
& \left(Q_{k}^{\top} P_{k+1}-\Delta\right) U_{k}^{\top} J=J U_{k}\left(P_{k+1}^{\top} Q_{k}-\Delta\right) \\
& \Rightarrow \quad\left(Q_{k}^{\top} P_{k}-\Delta U_{k}^{\top}\right) J=J\left(P_{k}^{\top} Q_{k}-U_{k} \Delta\right) \quad \text { using }(5.7) \\
& \Rightarrow \quad J U_{k} \Delta-\Delta U_{k}^{\top} J=J P_{k}^{\top} Q_{k}-Q_{k}^{\top} P_{k} J \\
& \Rightarrow \quad J\left(U_{k} \Delta-\Delta U_{k}^{-1}\right)=J\left(P_{k}^{\top} Q_{k}-\left(P_{k}^{\top} Q_{k}\right)^{-1}\right) \quad \text { since } U_{k} \in G \text { and } \Delta J=J \Delta .
\end{aligned}
$$

This gives us the relation

$$
U_{k} \Delta-\Delta U_{k}^{-1}=P_{k}^{\top} Q_{k}-\left(P_{k}^{\top} Q_{k}\right)^{-1},
$$

which is equivalent to (5.6) when $U_{k}$ is in the neighborhood of the identity in $G$ where $\Sigma_{D}^{-1}$ exists as given by Lemma 5.1 .

The above result generalizes Theorem 6.5 in [3], which gives the symmetric representation of the extremal flows of the discrete optimal control problem in $\mathrm{SO}(n)$, to the quadratic groups $G$. Equation (5.6) can be written as

$$
\Sigma_{D}\left(U_{k}\right)=P_{k}^{\top} Q_{k}-\left(P_{k}^{\top} Q_{k}\right)^{-1} .
$$

$\Sigma_{D}$ can be inverted to obtain $U_{k}$ from (5.9) as long as $U_{k}$ remains in a neighborhood of the identity. Therefore, the discrete optimal equations can be used in a computational routine to obtain the discrete extremal trajectory in $G \times G$, where we obtain $U_{k}$ given $\left(Q_{k}, P_{k}\right) \in G \times G$ using (5.6) and then use (5.5) to obtain $\left(Q_{k+1}, P_{k+1}\right)$. More generally in the Moser-Veselov setting of $G=\mathrm{SO}(n)$, the identity component of $\mathrm{O}(n)$, it is possible to find a global unique solution of $U_{k}$ from (5.9). For details see $[3,5,16]$ and also [6]. We now show that in the more general situation where $G$ is one of the quadratic groups (2.1), there are at most two possible solutions for $U_{k}$ when $\Sigma_{D}$ is invertible. For $G=\mathrm{O}(n)$, only one of these two solutions is in the identity component $(\mathrm{SO}(n))$, which accounts for the Moser-Veselov result mentioned above. 
5.2 Solutions for the discrete optimal control

Consider (5.9) for obtaining the optimal control $U_{k}$, and rewrite it as follows:

$$
U_{k} \Delta-\Delta U_{k}^{-1}=M_{k},
$$

where $M_{k}=P_{k}^{\top} Q_{k}-\left(P_{k}^{\top} Q_{k}\right)^{-1}$. From (5.5), we see that the sequence $\left\{M_{k}\right\}$ satisfies

$$
M_{k+1}=U_{k}^{-1} M_{k} U_{k}
$$

Equations (5.10) and (5.11) form the discrete version of the continuous generalized Euler equations (3.27). We are now concerned with the solution of this discrete system of equations.

Equation (5.10) is an algebraic equation of the form

$$
g \Delta-\Delta g^{-1}=A, \quad g \in G, A \in \mathfrak{g} .
$$

We first obtain from this equation an equivalent equation on $\mathfrak{g}$. Let $\kappa$ be a square root matrix of the positive definite matrix $\Delta$, i.e., $\Delta=\kappa^{2}$, so that $J \kappa=\kappa J$ and $\kappa=\kappa^{\top}$. Then from (5.12), we get

$$
\kappa^{-1} g \kappa-\kappa g^{-1} \kappa^{-1}=\kappa^{-1} A \kappa^{-1} .
$$

Substituting $g=\exp (X)$ where $X \in \mathfrak{g}$ in the above expression, we obtain

$$
\exp \left(\kappa^{-1} X \kappa^{-1} \Delta\right)-\exp \left(-\Delta \kappa^{-1} X \kappa^{-1}\right)=\kappa^{-1} A \kappa^{-1}
$$

Note that if $A \in \mathfrak{g}$, then $\kappa^{-1} A \kappa^{-1} \in \mathfrak{g}$, since

$$
\left(\kappa^{-1} A^{\top} \kappa^{-1}\right) J+J\left(\kappa^{-1} A \kappa^{-1}\right)=\kappa^{-1}\left(A^{\top} J+J A\right) \kappa^{-1}=0 .
$$

Then (5.13) becomes

$$
\exp (\bar{X} \Delta)-\exp (-\Delta \bar{X})=\bar{A},
$$

where $\bar{X}=\kappa^{-1} X \kappa^{-1}, \bar{A}=\kappa^{-1} A \kappa^{-1} \in \mathfrak{g}$. We can also verify that $X \in \mathfrak{g} \Rightarrow$ $\exp (X \Delta)-\exp (-\Delta X) \in \mathfrak{g}$, as shown below:

$$
\begin{aligned}
& (\exp (X \Delta)-\exp (-\Delta X))^{\top} J+J(\exp (X \Delta)-\exp (-\Delta X)) \\
& \quad=\left(\exp \left(\Delta X^{\top}\right)-\exp \left(-X^{\top} \Delta\right)\right) J+J(\exp (X \Delta)-\exp (-\Delta X)) \\
& \quad=\left(\exp \left(-\Delta J X J^{-1}\right)-\exp \left(J X J^{-1} \Delta\right)\right) J+J(\exp (X \Delta)-\exp (-\Delta X)) \\
& \quad=\left(\exp \left(-J \Delta X J^{-1}\right)-\exp \left(J X \Delta J^{-1}\right)\right) J+J(\exp (X \Delta)-\exp (-\Delta X)) \\
& \quad=J(\exp (-\Delta X)-\exp (X \Delta)) J^{-1} J+J(\exp (X \Delta)-\exp (-\Delta X))=0 .
\end{aligned}
$$

Therefore, (5.14) is indeed a system on $\mathfrak{g}$. If we define

$$
\sinh _{+} X \triangleq \frac{1}{2}(\exp (X \Delta)-\exp (-\Delta X))
$$


then $\sinh _{+}$is a mapping from $\mathfrak{g}$ to $\mathfrak{g}$. A solution of (5.14) is then obtained as

$$
\bar{X}=\sinh _{+}^{-1}\left(\frac{\bar{A}}{2}\right),
$$

and, therefore, a solution of (5.13) is given by

$$
X=\kappa \sinh _{+}^{-1}\left(\frac{1}{2}\left(\kappa^{-1} A \kappa^{-1}\right)\right) \kappa
$$

where $\sinh _{+}^{-1}$ is defined. This gives us a solution of the algebraic equation (5.12) as

$$
g_{1}=\exp \left(\kappa \sinh _{+}^{-1}\left(\frac{\bar{A}}{2}\right) \kappa\right) .
$$

We obtain a second solution of the algebraic equation (5.12) if we substitute $\hat{g}=$ $-g^{-1}$ or $g=-(\hat{g})^{-1}$ in this equation to get

$$
\Delta \hat{g}-(\hat{g})^{-1} \Delta=A .
$$

Further, substitute $\hat{g}=\exp (\hat{X}), \bar{X}=\kappa^{-1} X \kappa^{-1}$, and $\bar{A}=\kappa^{-1} A \kappa^{-1}$, in the above expression to obtain the equation below:

$$
\exp (\Delta \bar{X})-\exp (-\bar{X} \Delta)=\bar{A}
$$

Note that $\hat{g} \in G$, and hence $\hat{X}, \bar{X} \in \mathfrak{g}$. One can also similarly show that if $X \in \mathfrak{g}$ then $\exp (\Delta X)-\exp (-X \Delta) \in \mathfrak{g}$. Therefore, we can define the following mapping from $\mathfrak{g}$ to $\mathfrak{g}$ :

$$
\sinh _{-} X \triangleq \frac{1}{2}(\exp (\Delta X)-\exp (-X \Delta))
$$

We then obtain a solution of (5.18) as

$$
\bar{X}=\sinh _{-}^{-1}\left(\frac{\bar{A}}{2}\right),
$$

and, therefore, we get

$$
\hat{X}=\kappa \sinh _{-}^{-1}\left(\frac{1}{2}\left(\kappa^{-1} A \kappa^{-1}\right)\right) \kappa
$$

where $\sinh _{-}^{-1}$ is defined. This gives us the second solution of the algebraic equation (5.12) as

$$
g_{2}=-(\hat{g})^{-1}=-\exp \left(-\kappa \sinh _{-}^{-1}\left(\frac{\bar{A}}{2}\right) \kappa\right) .
$$

Thus we obtain two solutions to the algebraic equation (5.12), which are given by the inverse mappings of $\sinh _{+}$and $\sinh _{-}$. The mappings $\sinh _{+}$and $\sinh _{-}$are indeed 
different mappings on $\mathfrak{g}$, as can be seen from their expansions:

$$
\begin{aligned}
\sinh _{ \pm} X= & \frac{1}{2}(X \Delta+\Delta X) \pm \frac{1}{2} \frac{1}{2 !}\left((X \Delta)^{2}-(\Delta X)^{2}\right)+\frac{1}{2} \frac{1}{3 !}\left((X \Delta)^{3}+(\Delta X)^{3}\right) \\
& \pm \frac{1}{2} \frac{1}{4 !}\left((X \Delta)^{4}-(\Delta X)^{4}\right)+\cdots
\end{aligned}
$$

From these expansions and the definitions of the $\sinh _{+}$and $\sinh _{-}$maps, we note the following two properties:

(1) $-\sinh _{ \pm}(X)=\sinh _{\mp}(-X)$, and

(2) for $\Delta=\rho I$ (a positive multiple of the identity), all the even terms in the above expansions for $\sinh _{+}$and $\sinh _{-}$vanish and reduce to the usual series expression for sinh: i.e., $\sinh _{ \pm}(X)=\sinh (\rho X)$.

The first property shows how the $\sinh _{+}$and sinh_ mappings are related. From this property, we also get the relation between the inverse mappings, where these inverses exist; e.g., $-\sinh _{-}^{-1}(-A)=\sinh _{+}^{-1}(A)$. The second property leads to a simpler relation between the two solutions (5.17) and (5.21) of the algebraic equation, in the special case that $\Delta$ is a positive multiple of the identity matrix.

The case $\Delta=\rho I$ includes the case $G=\mathrm{O}(n)$. In this case, $A=\sinh _{+}(X)=$ $\sinh (\rho X)$ and

$$
\sinh _{+}^{-1}(A)=X=\frac{1}{\rho} \sinh ^{-1}(A) \Rightarrow \rho \sinh _{+}^{-1}=\sinh ^{-1} .
$$

Similarly, in this case, we can also show that

$$
\rho \sinh _{-}^{-1}=\sinh ^{-1} .
$$

Therefore, the two solutions of the algebraic equation (5.12) take the following forms:

$$
\begin{aligned}
& g_{1}=\exp \left(\rho \sinh _{+}^{-1}\left(\frac{\bar{A}}{2}\right)\right)=\exp \left(\sinh ^{-1}\left(\frac{A}{2 \rho}\right)\right), \\
& g_{2}=-\exp \left(-\rho \sinh _{-}^{-1}\left(\frac{\bar{A}}{2}\right)\right)=-\exp \left(-\sinh ^{-1}\left(\frac{A}{2 \rho}\right)\right)=-g_{1}^{-1},
\end{aligned}
$$

since $\bar{A}=\frac{1}{\rho} A$. Therefore, in this case, the algebraic equation (5.12) becomes

$$
g-g^{-1}=\frac{1}{\rho} A=\hat{g}-(\hat{g})^{-1},
$$

where $\hat{g}=-g^{-1}$, from which we can read of the solutions $g=g_{1}$ and $g=g_{2}=$ $-g_{1}^{-1}$ immediately. Note that if $G=\mathrm{O}(n)$, then one of the solutions is in the identity component $\mathrm{SO}(n)$, which is the solution described in [3].

Finally, we note that the system

$$
U_{k+1} \Delta-\Delta U_{k+1}^{-1}=M_{k+1}=U_{k}^{-1} M_{k} U_{k}
$$


therefore, has two possible solutions. These solutions are given by

$$
\begin{aligned}
U_{k+1} & =\exp \left(\kappa \sinh _{+}^{-1}\left(\frac{1}{2} \bar{M}_{k+1}\right) \kappa\right), \\
U_{k+1} & =-\exp \left(-\kappa \sinh _{-}^{-1}\left(\frac{1}{2} \bar{M}_{k+1}\right) \kappa\right) \\
& =-\exp \left(\kappa \sinh _{+}^{-1}\left(-\frac{1}{2} \bar{M}_{k+1}\right) \kappa\right),
\end{aligned}
$$

where $\bar{M}_{k+1}=\kappa^{-1} M_{k+1} \kappa^{-1}$. Note that the system $M_{k+1}=U_{k}^{-1} M_{k} U_{k}$ is isospectral. Therefore, the domain under which (5.22) is solvable for $U_{k+1}$ in a neighborhood of $0 \in \mathfrak{g}$ is the same as that for which (5.10) is solvable for $U_{k}$, if this domain is measured by a given bound on the spectral radius of $M_{k+1}=$ spectral radius of $M_{k}$. Note that this is exactly the domain where $\Sigma_{D}$ is invertible in (5.9), and, therefore, we can obtain $U_{k}$ from (5.6) in this domain.

\subsection{The Example of $\mathrm{Sp}(2)$}

For the two-dimensional symplectic group $G=\operatorname{Sp}(2)$, we have

$$
n=2, \quad \alpha=-1, \quad J=\left[\begin{array}{cc}
0 & 1 \\
-1 & 0
\end{array}\right] \text {. }
$$

In this case, it is easy to obtain the two solutions of the algebraic equation (5.12) explicitly when

$$
\Delta=\rho I=\left[\begin{array}{ll}
\rho & 0 \\
0 & \rho
\end{array}\right], \rho>0 .
$$

If $g \in \mathrm{Sp}(2)$, then one can verify that

$$
g=\left[\begin{array}{ll}
\alpha & \gamma \\
\delta & \beta
\end{array}\right], \quad \text { where } \alpha \beta-\delta \gamma=1
$$

Therefore, $\operatorname{Sp}(2)=\operatorname{SL}(2)$, the group of real $2 \times 2$ matrices with determinant 1 .

The Lie algebra $\mathfrak{s p}(2)=\mathfrak{s l}(2)$ of $\operatorname{Sp}(2)$ consists of real traceless $2 \times 2$ matrices. Therefore, (5.12) can be represented as the matrix equation

$$
\rho\left[\begin{array}{cc}
\alpha-\beta & 2 \gamma \\
2 \delta & \beta-\alpha
\end{array}\right]=\left[\begin{array}{cc}
a & c \\
b & -a
\end{array}\right]=A,
$$

so that $\gamma=\frac{c}{2 \rho}, \delta=\frac{b}{2 \rho}$, and $\alpha-\beta=\frac{a}{\rho}$. Since $\alpha \beta-\delta \gamma=1$, we have

$$
\beta\left(\beta+\frac{a}{\rho}\right)-\frac{c b}{4 \rho^{2}}=1 \quad \Rightarrow \quad \beta^{2}+\frac{a \beta}{\rho}-\frac{c b}{4 \rho^{2}}-1=0 .
$$


This gives us two possible solutions for $\beta$ and $\alpha$ :

$$
\begin{aligned}
& \beta=-\frac{a}{2 \rho} \pm \sqrt{\frac{a^{2}}{4 \rho^{2}}+\frac{c b}{4 \rho^{2}}+1}, \\
& \alpha=\frac{a}{2 \rho} \pm \sqrt{\frac{a^{2}}{4 \rho^{2}}+\frac{c b}{4 \rho^{2}}+1} .
\end{aligned}
$$

Using the fact that $\operatorname{det} A=-\left(a^{2}+c b\right)$, from the above expressions we obtain

$$
g=\frac{1}{2 \rho} A \pm I \sqrt{1-\frac{\operatorname{det} A}{4 \rho^{2}}} .
$$

Thus, (5.12) for $G=\operatorname{Sp}(2)$ has solution(s) if and only if $4 \rho^{2} \geq \operatorname{det} A$. Note that

$$
-g^{-1}=\left[\begin{array}{cc}
-\beta & \gamma \\
\delta & -\alpha
\end{array}\right]=\frac{1}{2 \rho} A \mp I \sqrt{1-\frac{\operatorname{det} A}{4 \rho^{2}}} .
$$

Hence, if $4 \rho^{2}>\operatorname{det} A$ there are two solutions of (5.12) given by (5.25), and the mapping $g \mapsto-g^{-1}$ permutes between these two solutions.

\section{Conclusions and Future Directions}

In this paper, we generalize the Euler equations and the symmetric representation of the $n$-dimensional rigid body equations to some quadratic matrix subgroups $G$ of the general linear groups $\operatorname{GL}(n)$. These matrix groups are defined through a quadratic matrix identity.

In addition, we describe the relationship between the symplectic structures on $G \times G$ and the cotangent bundle $T^{\star} G \simeq G \times \mathfrak{g}^{\star}$ and find symplectic submanifolds of $G \times G$ and $T^{\star} G$ that are invariant with respect to these extremal flows. Results on the discrete version of the symmetric representation of the extremal flows on $G \times G$ and on $G \times \mathfrak{g}^{\star}$, which generalize the Moser-Veselov equations to these quadratic groups, are also presented. These discrete results can be used as numerical algorithms to time-propagate the extremal flows. We also obtained some possible solutions to the optimal control for both the continuous and the discrete extremal flows.

Further developments on the discrete extremal equations, including bounds on the discrete momentum for which solutions to the discrete optimal control can be obtained are among future research plans in this area. We also intend to analyze the complete integrability, and explore Lax pair formulations in the setting of symmetric representations of these extremal flows.

In a future paper, we intend to work on an extension of this work to the special linear groups, and obtain the extremal flows of the optimal control problem on SL $(n)$. This would generalize both the Euler equations and the symmetric representation of the extremal flows in the quadratic groups $G$ to $\operatorname{SL}(n)$. Discrete versions of the 
extremal flows on $\operatorname{SL}(n)$ and their use as numerical algorithms to propagate these flows will also be investigated. These flows on the special linear group are interesting as models of deformable bodies and finite-dimensional models of fluids.

Acknowledgements The research reported in this paper was partially supported by the National Science Foundation. We would also like thank the referee whose suggestions greatly improved the exposition of this paper.

\section{References}

1. V.I. Arnold, Dynamical Systems III. Encyclopedia of Mathematics, vol. 3 (Springer, Berlin, 1988).

2. A. Bloch, J. Ballieul, P. Crouch, J.E. Marsden, Nonholonomic Mechanics and Control. Interdisciplinary Applied Mathematics, vol. 24 (Springer, Berlin, 2003).

3. A.M. Bloch, P. Crouch, J.E. Marsden, T.S. Ratiu, The symmetric representation of the rigid body equations and their discretization, Nonlinearity 15, 1309-1341 (2002).

4. A.M. Bloch, V. Brinzanescu, A. Iserles, J.E. Marsden, T.S. Ratiu, A class of integrable geodesic flows on the symplectic group, preprint (2007).

5. J. Cardoso, F. Leite, The Moser Veselov equation. Pre-Publicaos do Departamento de Matematic, Universidade de Coimbra (2001).

6. P. Deift, L.-C. Li, P. Tomei, Loop groups, discrete versions of some classical integrable systems, and rank 2 extensions, Mem. Am. Math. Soc. 100 (1992).

7. Y.N. Fedorov, Various aspects of $n$-dimensional rigid body dynamics, Am. Math. Soc. Trans. 168, 141-171 (1995).

8. I.M. Gelfand, S.V. Fomin, Calculus of Variations (Prentice Hall, New York, 1963) (reprinted by Dover, 2000).

9. G.H. Golub, C.F. Van Loan, Matrix Computations, 3rd edn. (Johns Hopkins University Press, Baltimore, 1996).

10. S. Helgason, Differential Geometry, Lie Groups and Symmetric Spaces (Academic, San Diego, 1978).

11. A. Jeffrey, Inverse Trigonometric and Hyperbolic Functions \$2.7, in Handbook of Mathematical Formulas and Integrals, 2nd edn. (Academic, Orlando, 2000), pp. 124-128.

12. D.E. Kirk, Optimal Control Theory: An Introduction (Dover, New York, 2004).

13. S.V. Manakov, Note on the integration of Euler's equations of the dynamics of an $n$-dimensional rigid body, Funct. Anal. Appl. 10, 253-299 (1976).

14. J.E. Marsden, T.S. Ratiu, Introduction to Mechanics and Symmetry, 2nd edn. Texts in Applied Mathematics, vol. 17 (Springer, Berlin, 1999).

15. A.S. Mischenko, A.T. Fomenko, Integrability of Euler equations on semisimple Lie algebras, Sel. Math. Sov. 2, 207-291 (1982).

16. J. Moser, A. Veselov, Discrete versions of some classical integrable systems and factorization of matrix polynomials, Commun. Math. Phys. 139, 217-243 (1991).

17. T. Ratiu, The motion of the free $n$-dimensional rigid body, Ind. Univ. Math. J. 29, 609-629 (1980).

18. V.S. Varadarajan, Lie Groups, Lie Algebras, and Their Representations (Springer, New York, 1984).

19. J.A. Wolf, Spaces of Constant Curvature, 2nd edn. (Publish or Perish, Boston, 1972).

20. H. Yoshimura, J.E. Marsden, Dirac structures in Lagrangian mechanics Part II: Variational structures, J. Geom. Phys. 57, 209-250 (2006). 